\title{
Subordinators which are infinitely divisible w.r.t. time: Construction, properties, and simulation of max-stable sequences and infinitely divisible laws
}

\author{
Jan-Frederik Mai and Matthias Scherer \\ Technische Universität München \\ Parkring 11, 85478 Garching-Hochbrück, Germany \\ E-mail address: mai@tum.de, scherer@tum.de
}

\begin{abstract}
The concept of a Lévy subordinator is generalized to a family of nondecreasing stochastic processes, which are parameterized in terms of two Bernstein functions. Whereas the independent increments property is only maintained in the Lévy subordinator special case, the considered family is always strongly infinitely divisible with respect to time, meaning that a path can be represented in distribution as a finite sum with arbitrarily many summands of independent and identically distributed paths of another process. Besides distributional properties of the process, we present two applications to the design of accurate and efficient simulation algorithms. First, each member of the considered family corresponds uniquely to an exchangeable max-stable sequence of random variables, and we demonstrate how the associated extreme-value copula can be simulated exactly and efficiently from its Pickands dependence measure. Second, we show how one obtains different series and integral representations for infinitely divisible probability laws by varying the parameterizing pair of Bernstein functions, without changing the law of one-dimensional margins of the process. As a particular example, we present an exact simulation algorithm for compound Poisson distributions from the Bondesson class, for which the generalized inverse of the distribution function of the associated Stieltjes measure can be evaluated accurately.
\end{abstract}

\section{Introduction}

We recall that a Lévy subordinator $L=\left\{L_{t}\right\}_{t>0}$ is a non-decreasing stochastic process on a probability space $(\Omega, \mathcal{F}, \mathbb{P})$ with independent and stationary increments, whose paths are almost surely right-continuous and start at $L_{0}=0$, see Bertoin (1999) for a textbook treatment. Intuitively, Lévy subordinators are

Received by the editors December 11th, 2018; accepted September 18th, 2019.

2010 Mathematics Subject Classification. 60G51, 60G70,60G09.

Key words and phrases. Strong infinitely divisible w.r.t. time, subordinator, infinitely divisible law, Pickands dependence function, Bondesson class, Bernstein function. 
the continuous-time analog of discrete-time random walks with non-negative increments. The law of $L$, that is its finite-dimensional distributions, is fully determined by the law of any random variable $L_{t}$ with $t>0$, whose Laplace transform is given by

$$
\mathbb{E}\left[e^{-x L_{t}}\right]=e^{-t \Psi_{L}(x)}, \quad \Psi_{L}(x)=\mu_{L} x+\int_{(0, \infty]}\left(1-e^{-u x}\right) \nu_{L}(\mathrm{~d} u), \quad x \geq 0,
$$

where the so-called Lévy measure $\nu_{L}$ satisfies $\int_{(0, \infty]} \min \{u, 1\} \nu_{L}(\mathrm{~d} u)<\infty$ and $\mu_{L} \geq 0$ is a drift constant. The function $\Psi_{L}$ is a so-called Bernstein function, see Schilling et al. (2010) for a textbook treatment, and the number $\nu(\{\infty\})$ is called the killing rate of $L$, because it corresponds to an exponential rate at which $L$ jumps to the absorbing graveyard state $\{\infty\}$, i.e. is "killed." The so-called Lévy-Khinchin formula (1.1) establishes a one-to-one correspondence between Bernstein functions and Lévy subordinators, so that $\Psi_{L}$ (or equivalently the pair $\left(\mu_{L}, \nu_{L}\right)$ ) provides a convenient analytical description of the law of $L$.

The purpose of the present article is to embed the concept of a Lévy subordinator into a larger family of non-decreasing processes that can be parameterized in terms of a pair $\left(\Psi_{L}, \Psi_{F}\right)$ of two Bernstein functions. On the one hand, the enlarged family of processes still satisfies the concept of being strongly infinitely divisible with respect to time, as explained below, which renders it a natural generalization from an algebraic viewpoint. On the other hand, our generalization is inspired by two practical applications: Firstly, the processes can be used to construct and simulate multivariate extreme-value distributions. Second, they provide a reasonable framework to derive series representations for infinitely divisible laws on the positive half-axis, which can be used for simulation.

For a pair $(F, L)$ of a distribution function $F$ of some non-negative random variable with finite, positive mean $\int_{0}^{\infty} 1-F(x) \mathrm{d} x \in(0, \infty)$ and a Lévy subordinator $L$ without drift, the present article studies distributional properties of the stochastic process

$$
H_{t}:=H_{t}^{(F, L)}=\int_{0}^{\infty}-\log \left\{F\left(\frac{s}{t}-\right)\right\} \mathrm{d} L_{s}, \quad t \geq 0,
$$

the integral being defined pathwise in the usual Riemann-Stieltjes sense, and with $F(x-):=\lim _{u \nearrow x} F(u)$. Since $F$ is right-continuous, so is $t \mapsto F(s / t-)$ for each fixed $s$. This implies that $t \mapsto H_{t}$ is almost surely right-continuous as integral over right-continuous functions. By definition, $H_{0}=0$ (using the notations $1 / 0:=\infty$ and $F(\infty-):=1)$, and $H_{t} \in[0, \infty]$ for $t \geq 0$, i.e. in particular $H_{t}=\infty$ is possible. An alternative representation for $H$ is given by the formula

$$
H_{t}=\int_{0}^{\infty} L_{s t} \mathrm{~d}(\log (F(s))), \quad t \geq 0
$$

which is a consequence of Tonelli's Theorem:

$$
\begin{aligned}
\int_{(0, \infty)} L_{s t} \mathrm{~d}(\log (F(s))) & =\int_{(0, \infty)} \int_{(0, s t]} \mathrm{d} L_{u} \mathrm{~d}(\log (F(s))) \\
& =\int_{(0, \infty)} \int_{[u / t, \infty)} \mathrm{d}(\log (F(s))) \mathrm{d} L_{u} \\
& =\int_{(0, \infty)}-\log \left\{F\left(\frac{s}{t}-\right)\right\} \mathrm{d} L_{u}=H_{t}
\end{aligned}
$$


According to Mansuy (2005, Paragraph (2.2)) this shows that $H=\left\{H_{t}\right\}_{t>0}$ is strongly infinitely divisible with respect to time (strong IDT) ${ }^{1}$, meaning that for arbitrary $n \in \mathbb{N}$ we have the distributional equality

$$
\left\{H_{t}\right\}_{t \geq 0} \stackrel{d}{=}\left\{\sum_{i=1}^{n} H_{\frac{t}{n}}^{(i)}\right\}_{t \geq 0},
$$

where $H^{(i)}, i=1, \ldots, n$, denote independent copies of $H$. In particular, $H_{t}$ is an infinitely divisible random variable and there exists a Bernstein function $\Psi_{H}$ such that $\exp \left(-t \Psi_{H}\right)$ equals the Laplace transform of $H_{t}$ for arbitrary $t \geq 0$.

We call a pair $(F, L)$ admissible, whenever $H_{t}$ is not almost surely equal to the trivial process $H_{t}=\infty \cdot \mathbf{1}_{\{t>0\}}$, which can happen in general. Our interest in this semi-parametric family of stochastic processes is fueled both by theoretical and practical aspects. In the following Section 2 we study distributional properties, whereas Sections 3 and 4 give applications of the presented class of processes to the design of simulation algorithms. More precisely, the following list outlines the organization of the remaining article and the contributions made.

Section 2: From the viewpoint of the theory on infinite divisibility, the considered family of stochastic processes is a natural extension of the concept of a Lévy subordinator. Lévy subordinators arise in the special case when $F$ corresponds to a Bernoulli distribution with success probability $\exp (-1)$, see Example 2.8. Strong IDT processes have first been introduced in Mansuy (2005) and further examples have been studied in Es-sebaiy and Ouknine (2008); Hakassou and Ouknine (2013). These references give some examples of strong IDT processes with an emphasis on Gaussian processes. A LePage series representation for strong IDT processes without Gaussian component, in particular for non-negative strong IDT processes, is derived in Kopp and Molchanov (2018), and has been refined in the non-decreasing case by Mai (2019). The present investigation demonstrates that the considered processes form a large, and particularly tractable, subfamily of non-decreasing strong IDT processes. In particular, its two parameter-"dimensions" $F$ and $L$ are explained to be dual in a certain sense, see Lemma 2.3 and Section 4. Regarding applications, in the particular case when $F$ is continuous and satisfies $F(1)=1$ the process $H$ is applied as a time-change in a model for stock prices in James and Zhang (2011), building upon earlier work of Bender and Marquardt (2009) who study a more general family of convoluted subordinators for the same purpose.

We demonstrate how several distributional properties of $H$ can be inferred conveniently from the parameterizing pair $(F, L)$, thus pave the way to an analytical treatment of $H$ via its parameters. In particular, in addition to the defining integral representation we present a canonical LePage series representation for $H$ in the spirit of Kopp and Molchanov (2018). We also present necessary and sufficient analytical conditions for the probability law of $H_{1}$ to be of compound Poisson type or to have an atom at $\infty$. Section 2.3 further studies the natural filtration $\left\{\mathcal{F}_{t}^{H}\right\}_{t \geq 0}$ of the process $H$. While Lévy subordinators have independent increments, we demonstrate how the support of the probability measure $\mathrm{d} F$ controls the ability of $H$ to "see into the future." In particular, for bounded support of $\mathrm{d} F$, the increment $H_{t+h}-H_{t}$ can be decomposed into a sum of one part that is measurable with respect to $\mathcal{F}_{t}^{H}$, and another part that is independent thereof.

\footnotetext{
${ }^{1}$ This property is called time-stable in Kopp and Molchanov (2018).
} 
Section 3: For each realization of $H$, the function $x \mapsto 1-\exp \left(-H_{x}\right), x \geq 0$, is the distribution function of some non-negative random variable. In other words, $1-\exp (-H)$ is a random variable taking values in the set of distribution functions of non-negative random variables. Due to Mai and Scherer (2014, Theorem 5.3), an iid sequence $\left\{Y_{k}\right\}_{k \in \mathbb{N}}$ of random samples drawn from this a priori simulated distribution function is an exchangeable min-stable exponential sequence, meaning that $\min \left\{Y_{1} / t_{1}, \ldots, Y_{d} / t_{d}\right\}$ has an exponential distribution, whose exponential rate we denote by

$$
\ell\left(t_{1}, \ldots, t_{d}, 0,0, \ldots\right) \in[0, \infty), \quad d \in \mathbb{N}, t_{1}, \ldots, t_{d} \geq 0 \text { (not all zero). }
$$

Equivalently, $\left\{1 / Y_{k}\right\}_{k \geq 1}$ is an exchangeable max-stable sequence. If $Y_{1}$ has unit mean, i.e. if $-\log \left(\mathbb{E}\left[\exp \left\{-H_{1}\right\}\right]\right)=1$, the associated function $\ell:[0, \infty)_{00}^{\mathbb{N}} \rightarrow$ $[0, \infty)$ is called a stable tail dependence function, defined on the set $[0, \infty)_{00}^{\mathbb{N}}$ of nonnegative sequences that are eventually zero. The stable tail dependence function uniquely characterizes the law of $\left\{Y_{k}\right\}_{k \in \mathbb{N}}$ and, equivalently, the law of $H$. Since min- (resp. max-) stability is closely related to multivariate extreme-value theory, an understanding of the law of $H$ is thus tantamount with the understanding of an associated family of multivariate extreme-value copulas, see Gudendorf and Segers (2010) for background on the subject. In particular, a simulation algorithm for the random vector $\left(Y_{1}, \ldots, Y_{d}\right)$ is equivalent to one for the associated extreme-value copula.

Section 3 shows how the random vector $\left(Y_{1}, \ldots, Y_{d}\right)$ can be simulated exactly. To this end, we make use of a simulation algorithm presented in Dombry et al. (2016), which requires to simulate from the so-called Pickands dependence measure associated with $\left(Y_{1}, \ldots, Y_{d}\right)$, a finite measure on the $d$-dimensional unit simplex. In the present situation, we demonstrate how this simulation can be achieved efficiently and accurately.

Section 4: Fixing $t=1$, the random variable $H_{1}$ has an infinitely divisible law on $[0, \infty]$, which is invariant with respect to many changes in the parameterizing pair $(F, L)$. This fact can be used to derive different series representations for the same infinitely divisible law from Definition (1.2), when either $L$ is of compound Poisson type or the support of $\mathrm{d} F$ is bounded. In spirit, this methodology is quite similar to seminal ideas in Bondesson (1982), who proposes alternative series representations for infinitely divisible laws on $\mathbb{R}$. Section 4 demonstrates how the $(F, L)$-parameterization of $H_{1}$ provides a very convenient setting to derive a simulation algorithm for distributions from the so-called Bondesson class, whenever the associated Stieltjes measure is given in a more convenient form than the Lévy measure. In fact, if $L$ is chosen as a compound Poisson subordinator with unit exponential jumps, the definition of $H_{1}$ defines a bijection between the Bondesson class and distribution functions $F$ having finite mean and left-end point of support equal to zero. This is to some extent analogous to traditional results for the case when $L$ is a standard Gamma process, in which case it is well known that the definition of $H_{1}$ defines a bijection between the so-called Thorin class and distribution functions $F$ satisfying a certain integrability condition, see James et al. (2008, Propositions 1.1 and 1.6).

As a final remark regarding our exposition in Section 4 we like to point out that our investigation of the law of $H_{1}$ is in general different from a series of seemingly similar articles studying so-called exponential functionals of Lévy processes. Only 
in the special case $F(x)=\exp (-\exp (-x))$ the random variable $H_{1}$ defines an exponential functional of a Lévy process. In this case, Behme (2015, Proposition 3.5) states that if the law of $L_{1}$ lies in the Bondesson family and satisfies $\mathbb{E}\left[\log \left(L_{1}\right)\right]<\infty$, then the law of $H_{1}$ lies in the Thorin class. Whereas exponential functionals of Lévy processes are defined in terms of integrands of the form $t \mapsto \exp \left(-\xi_{t}\right)$ for a Lévy process $\xi$, which might in general be different from the trivial process $\xi_{t}=t$, one crucial difference in the present investigation is that our integrand - albeit being always deterministic - needs not be of exponential form (i.e. we consider arbitrary $F$ and not only $F(x)=\exp (-\exp (-x)))$, so $H_{1}$ in general is not the exponential functional of a Lévy process.

Finally, Section 5 concludes.

\section{Anatomy of the process $H$}

2.1. Technical preliminaries and admissibility. Throughout, we denote by $L=$ $\left\{L_{t}\right\}_{t \geq 0}$ a (possibly killed) Lévy subordinator without drift and with Lévy measure $\nu_{L}$ on $(0, \infty]$, i.e. with killing rate $\nu(\{\infty\})$. We assume that $\nu_{L}$ is non-zero, i.e. $L$ is not identically zero. Its associated Bernstein function is denoted by

$$
\Psi_{L}(x):=\int_{(0, \infty]}\left(1-e^{-u x}\right) \nu_{L}(\mathrm{~d} u), \quad x \geq 0,
$$

implicitly using the short-hand notations $\exp (-\infty):=0$ and $0 \cdot \infty:=0$ in order to enforce $\Psi_{L}(0)=0$.

Remark 2.1 (Why only driftless $L$ are considered?). A positive drift $\mu_{L}$ of the Lévy subordinator $L$ would imply a drift of the process $H$, namely

$$
\mu_{H}=\mu_{L} \int_{0}^{\infty}-\log \{F(s)\} \mathrm{d} s .
$$

On the one hand, this is inconvenient, because it requires an additional integrability condition on $F$, so that (2.1) exists at all. We will always postulate that $F$ satisfies $\int_{0}^{\infty} 1-F(s) \mathrm{d} s<\infty$, which is a weaker condition. For instance, the distribution function $F(x)=\exp \left(-x^{-2}\right)$ may be admissible in the following but (2.1) is not finite. On the other hand, the assumption of a driftless Lévy subordinator is without loss of generality. To explain this, recall that $H$ falls into the family of non-decreasing strong IDT processes. It follows from a structural result in Mai (2019) that, just like for the subfamily of Lévy subordinators, such processes can be decomposed uniquely into $H_{t}=\mu_{H} t+\tilde{H}_{t}$, with a drift $\mu_{H} \geq 0$ and a nondecreasing strong IDT process $\tilde{H}$ without drift. This allows us to concentrate our study on the driftless case, because the more general case is simply obtained by adding a drift a posteriori.

For later reference, we introduce the following sets of distribution functions (cdfs)

$$
\begin{aligned}
\mathfrak{F}_{c} & :=\left\{F \text { cdf of some } X \in[0, \infty): c=\mathbb{E}[X]=\int_{0}^{\infty} 1-F(s) \mathrm{d} s\right\}, \\
\mathfrak{F} & :=\bigcup_{c \in(0, \infty)} \mathfrak{F}_{c} .
\end{aligned}
$$

The following result is derived in Mai (2018) and occupies a commanding role with regards to an analytic treatment of the stochastic properties of $H$, thus is repeated 
here for the sake of an educational exposition. In particular, it shows that the parameterizing pair $(F, L)$ may conveniently be characterized analytically in terms of two Bernstein functions $\Psi_{L}$ (characterizing the law of $L$ ) and $\Psi_{F}$ (characterizing F).

Lemma 2.2 (Bernstein functions associated with $\mathfrak{F}$ ). For $F \in \mathfrak{F}$ the function

$$
\Psi_{F}(x):=\int_{0}^{\infty} 1-F(s)^{x} \mathrm{~d} s, \quad x \geq 0,
$$

is a Bernstein function and the mapping $F \mapsto \Psi_{F}$ is a bijection between $\mathfrak{F}$ and the set of Bernstein functions without drift. The Lévy measure $\nu_{F}$ associated with $\Psi_{F}$ is determined in terms of $F$ by the equation $\nu_{F}((t, \infty])=F^{-1}(\exp (-t)), t>0$, where $F^{-1}$ denotes the generalized inverse of $F$. The inverse mapping $\nu \mapsto F_{\nu}$ from the set of Lévy measures on $(0, \infty]$ to $\mathfrak{F}$ is given by

$$
F_{\nu}(t)= \begin{cases}0 & , \text { if } t<\nu(\{\infty\}) \\ e^{-S_{\nu}^{-1}(t)} & , \text { if } \nu(\{\infty\}) \leq t<\nu((0, \infty]), \\ 1 & , \text { else }\end{cases}
$$

where $S_{\nu}^{-1}$ denotes the generalized inverse of $S_{\nu}(t):=\nu((t, \infty])$.

Proof: This is precisely Mai (2018, Lemma 3), where a detailed proof can be found. The basic idea is that under the mapping $u \mapsto-\log \{F(u)\}$ the Lebesgue measure on $(0, \infty)$ is mapped to a measure $\nu_{F}$ and the integral (2.2) defining $\Psi_{F}$ becomes

$$
\Psi_{F}(x)=\int_{(0, \infty]} 1-e^{-u x} \nu_{F}(\mathrm{~d} u) .
$$

The assumption $F \in \mathfrak{F}$ is equivalent to integrability of $1-F$, which is equivalent to $\int_{(0, \infty]} \min \{x, 1\} \nu_{F}(\mathrm{~d} x)<\infty$, showing that $\nu_{F}$ is a Lévy measure and hence $\Psi_{F}$ a Bernstein function.

We always assume that $F$ in the definition of $H$ lies in $\mathfrak{F}$, and it is convenient to study the law of $H$ in terms of the function

$$
\ell(\vec{t}):=-\log \left(\mathbb{E}\left[e^{-\sum_{k \geq 1} H_{t_{k}}}\right]\right), \quad \vec{t} \in[0, \infty)_{00}^{\mathbb{N}} .
$$

Furthermore, the Laplace exponent of the infinitely divisible random variable $H_{1}$ is denoted by $\Psi_{H}$ in the sequel, i.e.

$$
\Psi_{H}(x):=-\log \left(\mathbb{E}\left[e^{-x H_{1}}\right]\right), \quad x \geq 0 .
$$

Lemma 2.3 (Stable tail dependence function associated with $H$ ). Let $F \in \mathfrak{F}$ and $L$ be a driftless, non-zero Lévy subordinator. Then

$$
\ell(\vec{t})=\int_{(0, \infty]} \int_{0}^{\infty}\left(1-\prod_{k \geq 1} F\left(\frac{s}{t_{k}}\right)^{y}\right) \mathrm{d} s \nu_{L}(\mathrm{~d} y), \quad \vec{t} \in[0, \infty)_{00}^{\mathbb{N}} .
$$

Furthermore, we have the following expressions for the Bernstein function $\Psi_{H}$, which indicate a duality between the parameters $L$ and $F$ :

$$
\begin{aligned}
\Psi_{H}(x) & =\int_{(0, \infty]} \Psi_{F}(x y) \nu_{L}(\mathrm{~d} y)=\int_{(0, \infty]} \Psi_{L}(x y) \nu_{F}(\mathrm{~d} y) \\
& =\int_{(0, \infty]} \Psi_{L}\left(-\log \left\{F(u)^{x}\right\}\right) \mathrm{d} u
\end{aligned}
$$


Proof: Writing out the Riemann-Stieltjes definition of $H$, introducing for $N \in \mathbb{N}$ and $R \gg 1$ the notation

$$
s_{n, N}^{(R)}:=\frac{1}{R}+\frac{n\left(R-\frac{1}{R}\right)}{N}, \quad n=0, \ldots, N,
$$

we have

$$
\begin{aligned}
H_{t} & =\lim _{R \rightarrow \infty} \int_{\frac{1}{R}}^{R}-\log \left\{F\left(\frac{s}{t}-\right)\right\} \mathrm{d} L_{s} \\
& =\lim _{R \rightarrow \infty} \lim _{N \rightarrow \infty} \sum_{n=1}^{N}-\log \left\{F\left(\frac{s_{n-1, N}^{(R)}}{t}-\right)\right\}\left(L_{s_{n, N}^{(R)}}-L_{s_{n-1, N}^{(R)}}\right) .
\end{aligned}
$$

Using the bounded convergence theorem in $(*)$ and the stationary and independent increment property of $L$ in (**) implies

$$
\begin{aligned}
\mathbb{E}\left[e^{-\sum_{k=1}^{d} H_{t_{k}}}\right] & =\mathbb{E}\left[\exp \left\{-\lim _{R \rightarrow \infty} \lim _{N \rightarrow \infty} \sum_{n=1}^{N}\left(L_{s_{n, N}^{(R)}}-L_{s_{n-1, N}^{(R)}}\right) \sum_{k=1}^{d}-\log \left[F\left(\frac{s_{n-1, N}^{(R)}}{t_{k}}-\right)\right]\right\}\right] \\
& \stackrel{(*)}{=} \lim _{R \rightarrow \infty} \lim _{N \rightarrow \infty} \mathbb{E}\left[\exp \left\{-\sum_{n=1}^{N}\left(L_{s_{n, N}^{(R)}}-L_{s_{n-1, N}^{(R)}}\right) \sum_{k=1}^{d}-\log \left[F\left(\frac{s_{n-1, N}^{(R)}}{t_{k}}-\right)\right]\right\}\right] \\
& \stackrel{(* *)}{=} \lim _{R \rightarrow \infty} \lim _{N \rightarrow \infty} \prod_{n=1}^{N} \mathbb{E}\left[\exp \left\{-L_{\frac{R-\frac{1}{R}}{N}} \sum_{k=1}^{d}-\log \left[F\left(\frac{s_{n-1, N}^{(R)}}{t_{k}}-\right)\right]\right\}\right] \\
& =\lim _{R \rightarrow \infty} \lim _{N \rightarrow \infty} \prod_{n=1}^{N} \exp \left\{-\frac{R-\frac{1}{R}}{N} \int_{(0, \infty]}\left(1-\prod_{k=1}^{d} F\left(\frac{s_{n-1, N}^{(R)}}{t_{k}}-\right)^{u}\right) \nu_{L}(\mathrm{~d} u)\right\} \\
& =\lim _{R \rightarrow \infty} \exp \left\{-\int_{\frac{1}{R}}^{R} \int_{(0, \infty]}\left(1-\prod_{k=1}^{d} F\left(\frac{s}{t_{k}}-\right)^{u}\right) \nu_{L}(\mathrm{~d} u) \mathrm{d} s\right\} \\
& =\exp \left\{-\int_{0}^{\infty} \int_{(0, \infty]}^{d}\left(1-\prod_{k=1}^{d} F\left(\frac{s}{t_{k}}-\right)^{u}\right) \nu_{L}(\mathrm{~d} u) \mathrm{d} s\right\} .
\end{aligned}
$$

The order of the two remaining integrations can be switched by Tonelli's Theorem. When integrating with respect to $\mathrm{d} s$, it is further possible to change from $F(s-)$ to $F(s)$, since the at most countably many jump times of $F$ play no role in the integration.

By a completely analogous computation we obtain the equality

$$
\Psi_{H}(x)=\int_{(0, \infty]} \Psi_{F}(x y) \nu_{L}(\mathrm{~d} y) .
$$

Alternatively, (2.3) also follows for $x \in \mathbb{N}$ from the formula for $\ell$ in the special case $t_{1}=\ldots=t_{x}=1$ (and all other entries of $\vec{t}$ equal to zero) and for general $x \geq 0$ by the facts that (i) the right-hand side of (2.3) defines a Bernstein function by Schilling et al. (2010, Corollary 3.7) and (ii) a Bernstein function is uniquely determined by its values on $\mathbb{N}$, see Gnedin and Pitman (2008, p. 36). That the roles of $L$ and $F$ can be switched in (2.3) is an obvious consequence of Tonelli's Theorem. The last claimed representation is a consequence of the change of measure in the proof of Lemma 2.2. 
We view $\ell$ as a mapping from the set $[0, \infty)_{00}^{\mathbb{N}}$ of sequences which are eventually zero to $[0, \infty]$. The substitution $u=s / r$ shows that $\ell$ is homogeneous of order one, that is $\ell(r \cdot \vec{t})=r \ell(\vec{t})$ for $r \geq 0$ and $\vec{t} \in[0, \infty)_{00}^{\mathbb{N}}$. The mapping $\ell$ specifies the law of $H$ uniquely, i.e. its finite-dimensional distributions. This is due to the fact that the law of the random vector $\left(H_{t_{1}}, \ldots, H_{t_{d}}\right)$ is uniquely determined by the values of its multivariate Laplace transform on $\mathbb{N}^{d}$, which follows from the Stone-Weierstrass Theorem (polynomials are dense in the space of continuous functions on $[0,1]^{d}$ ). Consequently, the mapping $\ell$, which in turn by Lemma 2.3 is specified by $F$ and $\nu_{L}$, is a convenient analytical description for the law of $H$.

The condition $\mathbb{E}[X]>0$ in the definition of $\mathfrak{F}$ implies $F(0)<1$, which results in $\lim _{t \rightarrow \infty} H_{t}=\infty$ a.s.. The condition $\mathbb{E}[X]<\infty$ in the definition of $\mathfrak{F}$ is necessary (but needs not be sufficient) to have $\mathbb{P}\left(H_{t}<\infty\right)>0$ for $t>0$, i.e. necessary for admissibility. In order to explain this, Lemma 2.2 implies in particular that the integral $\int_{0}^{\infty} 1-F(s)^{x} \mathrm{~d} s$ is finite for all $x>0$ if and only if it is finite for a single fixed $x>0$. Now $\mathbb{P}\left(H_{t}<\infty\right)>0$ is equivalent to $\ell(t, 0,0, \ldots)<\infty$ and from Lemma 2.3 we see that this necessarily requires $\int_{0}^{\infty} 1-F(s)^{y} \mathrm{~d} s$ to be finite for those $y$ on which $\nu_{L}(\mathrm{~d} y)$ puts mass, which must be somewhere by the assumption $\nu_{L} \neq 0$. Hence, in particular $\mathbb{E}[X]=\int_{0}^{\infty} 1-F(s) \mathrm{d} s<\infty$.

While the condition $\mathbb{E}[X]<\infty$ in the definition of $\mathfrak{F}$ is necessary to prevent the non-interesting case $H_{t}=\infty \cdot \mathbf{1}_{\{t>0\}}$, we have claimed that this needs not be sufficient but depends on the specific choice of $L$ and $F$. To this end, we introduce the set

$$
\mathfrak{F}_{L}:=\left\{F \in \mathfrak{F}: \Psi_{H}(1)=\int_{(0, \infty]} \Psi_{F}(y) \nu_{L}(d y)<\infty\right\}
$$

of $L$-admissible distribution functions.

Lemma 2.4 (Admissibility). The following statements are equivalent.

(a) $(F, L)$ is admissible, i.e. $\mathbb{P}\left(H_{t}<\infty\right)>0$ for some (hence all) $t>0$.

(b) $F \in \mathfrak{F}_{L}$, i.e. $\Psi_{H}(1)<\infty$.

(c) $\ell(\vec{t})<\infty$ for arbitrary $\vec{t} \in[0, \infty)_{00}^{\mathbb{N}}$.

Proof: The equivalence of $(a)$ and $(b)$ is obvious, as well as the fact that $(c)$ implies (a) and (b). The only non-obvious statement is that admissibility in (a) implies (c). Denote the biggest argument of $\vec{t}$ by $t_{[d]}:=\max \left\{t_{1}, t_{2}, \ldots\right\}$ and $d:=\max \{n \in$ $\left.\mathbb{N}: t_{n}>0\right\}$. We compute

$$
\begin{aligned}
\ell(\vec{t}) & \leq \int_{(0, \infty]} \int_{0}^{\infty}\left(1-F\left(\frac{s}{t_{[d]}}\right)^{d y}\right) \mathrm{d} s \nu_{L}(\mathrm{~d} y) \\
& =t_{[d]} \int_{(0, \infty]} \Psi_{F}(d y) \nu_{L}(\mathrm{~d} y) \leq t_{[d]} d \int_{(0, \infty]} \Psi_{F}(y) \nu_{L}(\mathrm{~d} y) \stackrel{(*)}{<} \infty,
\end{aligned}
$$

where $(*)$ follows from $(L$-)admissibility and the above inequality from the estimate $g(d y) \leq d g(y)$, which holds for any concave function $g$ on $[0, \infty)$ with $g(0)=0$ and $d \in \mathbb{N}$, such as $g=\Psi_{F}$. To see this, for $d \in \mathbb{N}$, concavity and $g(0)=0$ imply that

$$
d g(y)=d g\left(\frac{d-1}{d} \cdot 0+\frac{1}{d} d y\right) \geq d\left(\frac{d-1}{d} g(0)+\frac{1}{d} g(d y)\right)=g(d y) .
$$

Remark $2.5\left(\nu_{L}\right.$ non-finite $\left.\Rightarrow \nu_{F}(\{\infty\})=0\right)$. If $(F, L)$ is admissible and $\nu_{L}$ is non-finite, then necessarily $\nu_{F}(\{\infty\})=0$, which is briefly explained. Since $\nu_{L}$ is 
non-finite, we have $\Psi_{L}(\infty):=\lim _{x \rightarrow \infty} \Psi_{L}(x)=\infty$. Admissibility implies with the help of Lemma 2.3 that

$$
\infty>\Psi_{H}(1)=\int_{(0, \infty]} \Psi_{L}(y) \nu_{F}(\mathrm{~d} y) \geq \Psi_{L}(\infty) \nu_{F}(\{\infty\})=\infty \nu_{F}(\{\infty\}),
$$

thus necessarily $\nu_{F}(\{\infty\})=0$.

Lemma 2.6 (Simple admissibility criteria).

(a) Let $F \in \mathfrak{F}$ and $L$ a non-zero Lévy subordinator without drift. If $\Psi_{F}$ is continuous at zero, bounded, and satisfies $\lim _{x \downarrow 0} \Psi_{F}^{\prime}(x)<\infty$, the pair $(F, L)$ is admissible, no matter how $L$ is chosen.

(b) Let $L$ be a (driftless) compound Poisson subordinator with intensity $\beta>0$ and jump size distribution $\mathbb{P}(J \in \mathrm{d} y)$, where $J$ denotes a generic jump size random variable on $(0, \infty]$. Then

$$
\mathfrak{F}_{L}=\left\{F \in \mathfrak{F}: \mathbb{E}\left[\Psi_{F}(J)\right]<\infty\right\} .
$$

In particular, if $\mathbb{E}[J]<\infty$ we have $\mathfrak{F}_{L}=\mathfrak{F}$.

Proof:

(a) By the assumptions on $\Psi_{F}$ there exists $c>0$ such that $\Psi_{F}(x) \leq c \min \{x, 1\}$ for all $x \geq 0$. We thus observe

$$
\Psi_{H}(1)=\int_{(0, \infty]} \Psi_{F}(x) \nu_{L}(\mathrm{~d} x) \leq c \int_{(0, \infty]} \min \{x, 1\} \nu_{L}(\mathrm{~d} x)<\infty .
$$

(b) The claim follows from $\nu_{L}(\mathrm{~d} y)=\beta \mathbb{P}(J \in \mathrm{d} y)$ and the fact that $\Psi_{F}(x) \leq$ $x \Psi_{F}(1)$ for $x \geq 1$ by concavity of $\Psi_{F}$.

2.2. Distributional properties. Lemma 2.7 derives an alternative stochastic representation of the process $\left\{H_{t}\right\}_{t \geq 0}$. This representation constitutes a particular special case of Kopp and Molchanov (2018, Theorem 4.2). To this end, recall from Lemma 2.4 that for an admissible pair $(F, L)$ the measure $\Psi_{F}(z) \nu_{L}(\mathrm{~d} z) / \Psi_{H}(1)$ defines a probability measure on $(0, \infty]$.

Lemma 2.7 (LePage series representation). Let $(F, L)$ be an admissible pair, i.e. $F \in \mathfrak{F}_{L}$. Let $\left\{Z_{k}\right\}_{k \geq 1}$ be an iid sequence drawn from the probability measure $\frac{\Psi_{F}(z)}{\Psi_{H}(1)} \nu_{L}(\mathrm{~d} z)$. Independently, let $\left\{\epsilon_{k}\right\}_{k \geq 1}$ be an iid sequence of unit exponential random variables. We then have the following equality in distribution:

$$
\left\{H_{t}\right\}_{t \geq 0} \stackrel{d}{=}\left\{-\sum_{k \geq 1} \log \left[F\left(\frac{\left(\epsilon_{1}+\ldots+\epsilon_{k}\right) \Psi_{F}\left(Z_{k}\right)}{t \Psi_{H}(1)}-\right)^{Z_{k}}\right]\right\}_{t \geq 0} .
$$

Proof: Denoting by $\delta_{(x, z)}$ the Dirac measure at a point $(x, z)$ in the plane, we note that $P:=\sum_{k \geq 1} \delta_{\left(\epsilon_{1}+\ldots+\epsilon_{k}, Z_{k}\right)}$ is a Poisson random measure on $[0, \infty) \times(0, \infty]$ with mean measure $\mathrm{d} x \times\left(\frac{\Psi_{F}(z)}{\Psi_{H}(1)} \nu_{L}(\mathrm{~d} z)\right)$ by Resnick (1987, Proposition 3.8). Consequently, denoting the stochastic process on the right-hand side of the claim by 
$\left\{\tilde{H}_{t}\right\}_{t \geq 0}$, the Laplace functional formula for Poisson random measure Resnick (1987, Proposition 3.6) yields

$$
\begin{aligned}
\mathbb{E}\left[e^{-\sum_{j \geq 1} \tilde{H}_{t_{j}}}\right] & =\exp \left(-\int_{(0, \infty]} \int_{0}^{\infty}\left(1-\prod_{j \geq 1} F\left(\frac{x}{t_{j}}-\right)^{z}\right) \mathrm{d} x \nu_{L}(\mathrm{~d} z)\right) \\
& =e^{-\ell(\vec{t})}=\mathbb{E}\left[e^{-\sum_{j \geq 1} H_{t_{j}}}\right]
\end{aligned}
$$

for arbitrary $\vec{t} \in[0, \infty)_{00}^{\mathbb{N}}$, establishing the claim.

Example 2.8 (The special case of a Lévy subordinator). Suppose that $F(x)=$ $\exp (-1)+(1-\exp (-1)) \mathbf{1}_{[1, \infty)}(x)$ is the cdf of a Bernoulli distribution with associated Bernstein function

$$
\Psi_{F}(x)=\int_{0}^{\infty} 1-F(s)^{x} \mathrm{~d} s=1-e^{-x}, \quad x \geq 0 .
$$

An arbitrary (driftless) Lévy subordinator $L$ leads to an admissible pair $(F, L)$ by Lemma 2.6 and obviously we have $H=L$. So Lemma 2.7 provides an infinite series representation for an arbitrary Lévy subordinator $L$, namely

$$
\left\{L_{t}\right\}_{t \geq 0}=\left\{\sum_{k \geq 1} Z_{k} \mathbf{1}_{\left\{\left(\epsilon_{1}+\ldots+\epsilon_{k}\right) \frac{1-e^{-Z_{k}}}{\Psi_{L}(1)} \leq t\right\}}\right\}_{t \geq 0} .
$$

In the special case when $L=N$ is a standard (unit intensity) Poisson process, this formula boils down to the well-known counting process representation

$$
\left\{N_{t}\right\}_{t \geq 0}=\left\{\sum_{k \geq 1} \mathbf{1}_{\left\{\epsilon_{1}+\ldots+\epsilon_{k} \leq t\right\}}\right\}_{t \geq 0}
$$

Representation (2.4) is a quite natural generalization of (2.5), and by Lemma 2.7 it is general enough to comprise all Lévy subordinators. In particular, it is worth mentioning that the probability law of the $Z_{k}$ is $(1-\exp (-z)) \nu_{L}(\mathrm{~d} z) / \Psi_{L}(1)$, which can be any probability law on $(0, \infty]$. This means that, conversely, if $\rho$ is an arbitrary probability law on $(0, \infty]$ and $\left\{Z_{k}\right\}_{k \geq 1}$ is an iid sequence drawn from $\rho$, (2.4) defines a Lévy subordinator $L$ without drift and with associated Lévy measure $\nu_{L}(\mathrm{~d} z)=(1-\exp (-z))^{-1} \rho(\mathrm{d} z)$.

Remark 2.9 (Series representation for ID $[0, \infty]$ ). Example 2.8 shows that Lemma 2.7 provides a series representation for infinitely divisible laws on $[0, \infty]$ (abbreviated $\operatorname{ID}[0, \infty]$ in the sequel), namely

$$
\left.L_{1} \stackrel{d}{=} \sum_{k \geq 1} Z_{k} \mathbf{1}_{\left\{\left(\epsilon_{1}+\ldots+\epsilon_{k}\right)\right.}\left(1-e^{-Z_{k}}\right) \leq \Psi_{L}(1)\right\}
$$

where $\left\{Z_{k}\right\}_{k \geq 1}$ is an iid sequence distributed according to the probability measure $\left(1-e^{-z}\right) \nu_{L}(\mathrm{~d} z) / \Psi_{L}(1)$, independent of $\left\{\epsilon_{k}\right\}_{k \geq 1}$. This representation is always an infinite series, even when $L_{1}$ has a compound Poisson distribution.

Recall that an element of $\operatorname{ID}[0, \infty]$ is of compound Poisson type if its associated Bernstein function $\Psi$ is bounded, i.e. $\lim _{x \rightarrow \infty} \Psi(x)<\infty$.

Lemma 2.10 (When does $H_{t}$ have a compound Poisson distribution?). Let $(F, L)$ be admissible. The Bernstein function $\Psi_{H}$ is bounded if and only if the following two conditions are satisfied:

(a) $\Psi_{L}$ is bounded, i.e. $L$ is a compound Poisson subordinator. 
(b) $\Psi_{F}$ is bounded, i.e. the random variable $X \sim F$ has bounded support.

Proof: Recall from Lemma 2.3 that $\Psi_{H}$ may be represented as

$$
\Psi_{H}(x)=\int_{0}^{\infty} \Psi_{L}\left(-\log \left(F(s)^{x}\right)\right) \mathrm{d} s .
$$

The sufficiency of the conditions (a) and (b) is clear from this representation, since $\Psi_{L}$ is bounded and the improper integral $\int_{0}^{\infty}[\ldots] \mathrm{d} s$ is actually a finite integral $\int_{0}^{u_{F}}[\ldots] \mathrm{d} s$, where $u_{F}$ is the right-end point of the support of $\mathrm{d} F$. Necessity is more difficult to observe. To this end, assume that $\Psi_{L}$ is unbounded. There is an $\epsilon>0$ and a $\delta>0$ such that $f(z):=-\log (F(z))>\delta$ for $z \in(0, \epsilon)$ by the condition $\mathbb{E}[X]>0$ in the definition of $\mathfrak{F}$. Consequently,

$$
\Psi_{H}(x) \geq \int_{0}^{\epsilon} \Psi_{L}(x \delta) \mathrm{d} s=\Psi_{L}(x \delta) \epsilon
$$

is obviously unbounded. Hence, we already see that $\Psi_{L}$ needs to be bounded. In this case, $L$ is of compound Poisson type, i.e. there is a Laplace transform $\varphi$ of a positive random variable on $(0, \infty]$ and a number $\beta>0$ such that $\Psi_{L}=\beta(1-\varphi)$. Consequently, we observe from (2.6) that

$$
\Psi_{H}(x)=\beta \int_{0}^{\infty}(1-\varphi(x f(s))) \mathrm{d} s .
$$

By assumption, we know that $\Psi_{H}$ is bounded, so we see with the help of Fatou's Lemma that

$$
\begin{aligned}
\infty & >\lim _{x \rightarrow \infty} \Psi_{H}(x)=\lim _{x \rightarrow \infty} \beta \int_{0}^{\infty}(1-\varphi(x f(s))) \mathrm{d} s \\
& \geq \beta \int_{0}^{\infty}\left(1-\liminf _{x \rightarrow \infty} \varphi(x f(s))\right) \mathrm{d} s .
\end{aligned}
$$

But $\varphi$ tends to zero as $u \rightarrow \infty$, since it is the Laplace transform of a positive random variable. Consequently, the assumption $f(z)>0$ for arbitrarily large $z>0$ leads to the contradiction $\infty>\infty$. Rather, we must have that $f(z)=0$ for $z \geq T$ with some finite $T$, which turns the last inequality into

$$
\infty>\beta \int_{0}^{T} 1 \mathrm{~d} s=\beta T,
$$

and is not a contradiction.

Recall that an element of $\operatorname{ID}[0, \infty]$ is said to have killing, if it assigns positive mass to $\{\infty\}$, which is the case if and only if its associated Lévy measure $\nu$ satisfies $\nu(\{\infty\})>0$. In terms of the associated Bernstein function $\Psi$, this means $\Psi(x)>$ $\epsilon>0$ for all positive $x>0$. In this case, we also say that the Bernstein function $\Psi$ has killing.

Lemma 2.11 (When does $H_{t}$ have a positive killing rate?). Let $(F, L)$ be admissible. The Bernstein function $\Psi_{H}$ has killing if and only if at least one of the following two conditions is satisfied:

(a) $\Psi_{F}$ has killing, i.e. the left end point of the support of $X \sim F$ is strictly positive.

(b) $\Psi_{L}$ has killing, i.e. $\nu_{L}(\{\infty\})>0$.

In this case, if $\Psi_{F}$ (resp. $\Psi_{L}$ ) has killing, then necessarily $\nu_{L}$ (resp. $\nu_{F}$ ) is finite. 
Proof: Sufficiency of the conditions (a) and (b): By Lemma 2.2, $\Psi_{F}$ has killing if and only if the left-end point of the support of $X \sim F$ is strictly positive. In this case, $\Psi_{F}(x)>\epsilon>0$ for all $x>0$ and one immediately observes from (2.3) that $H$ has killing in this case (since $L$, hence $\nu_{L}$, is non-zero by assumption) and $\nu_{L}$ is finite. Also, if $\Psi_{L}$ has killing, $\Psi_{H}(x)>\epsilon>0$ from Lemma 2.3 for all $x>0$, hence $H$ has killing and $\nu_{F}$ is finite.

Necessity of the conditions (a) and (b): Assume that neither (a) nor (b) holds. Equation (2.3) can be re-written as

$$
\begin{aligned}
\Psi_{H}(x) & =\int_{(0, \infty]} \int_{(0, \infty]}\left(1-e^{-x u s}\right) \nu_{F}(\mathrm{~d} s) \nu_{L}(\mathrm{~d} u) \\
& =\int_{(0, \infty)} \int_{(0, \infty)}\left(1-e^{-x u s}\right) \nu_{F}(\mathrm{~d} s) \nu_{L}(\mathrm{~d} u),
\end{aligned}
$$

where the last equality follows from the assumptions that $\nu_{L}(\{\infty\})=\nu_{F}(\{\infty\})=0$. Taking the limit as $x \searrow 0$ on both sides of this equation, and using the bounded convergence theorem, it follows that $\lim _{x \searrow_{0}} \Psi_{H}(x)=0$, so $H$ has no killing.

Since we are only interested in a description of the probability law of $H=H^{(F, L)}$, it is helpful to briefly ponder on potential redundancies, i.e. to investigate the question: When do two different pairs $(F, L) \neq(\tilde{F}, \tilde{L})$ lead to exactly the same probability law of the associated processes $H$ ? To address this issue in a mathematically rigorous manner, we introduce the equivalence relation

$$
(F, L) \sim(\tilde{F}, \tilde{L}): \Leftrightarrow \text { the law of } H^{(F, L)} \text { equals that of } H^{(\tilde{F}, \tilde{L})} .
$$

The equivalence class of an admissible pair $(F, L)$ is denoted by $[F, L]$, or also by $\left[F, \Psi_{L}\right]$, in the following. Unfortunately, we did not accomplish to find a convenient analytical description of the equivalence class $[F, L]$ in general, so this remains an interesting open problem. It is not difficult, however, to see that for an admissible pair $(F, L)$ the equivalence class $\left[F\left(c_{1} .\right)^{c_{2}}, c_{1} \Psi_{L}\left(. / c_{2}\right)\right]$ is invariant with respect to $c_{1}, c_{2}>0$. Depending on the admissible pair, however, there can be even more redundancies, and the following example demonstrates this quite extremely.

Example 2.12 (The curious case $F=$ Fréchet distribution). With a parameter $\theta \in(0,1)$ and $c_{\theta}:=\Gamma(1-\theta)^{-1 / \theta}$ consider the Fréchet distribution function $F(x):=$ $\exp \left(-c_{\theta} x^{-1 / \theta}\right) \mathbf{1}_{\{x>0\}}$ and observe that $\Psi_{F}(x)=x^{\theta}$. For arbitrary $y>0$ it is not difficult to compute

$$
\int_{0}^{\infty}\left(1-\prod_{k \geq 1} F\left(\frac{s}{t_{k}}\right)^{y}\right) \mathrm{d} s=y^{\theta}\left(\sum_{k \geq 1} t_{k}^{\frac{1}{\theta}}\right)^{\theta}, \quad \vec{t} \in[0, \infty)_{00}^{\mathbb{N}} .
$$

Let $L$ be a Lévy subordinator (without drift) and such that $F \in \mathfrak{F}_{L}$, i.e. such that $\Psi_{H}(1)=\int_{(0, \infty]} y^{\theta} \nu_{L}(\mathrm{~d} y)<\infty$. It follows that

$$
\ell(\vec{t})=\int_{(0, \infty]} y^{\theta} \nu_{L}(\mathrm{~d} y)\left(\sum_{k \geq 1} t_{k}^{\frac{1}{\theta}}\right)^{\theta}=\Psi_{H}(1)\left(\sum_{k \geq 1} t_{k}^{\frac{1}{\theta}}\right)^{\theta} .
$$

Consequently, the function $\ell$, hence the law of $H$, depends on the choice of $L$ only via the scalar $\Psi_{H}(1)$. In particular, we have

$$
\left[F, \Psi_{L}\right]=\left\{(F, \tilde{L}): c=\int_{(0, \infty]} y^{\theta} \nu_{\tilde{L}}(\mathrm{~d} y)<\infty\right\}
$$


for arbitrary $L$ satisfying $c=\int_{(0, \infty]} y^{\theta} \nu_{L}(\mathrm{~d} y)<\infty$, so in order to study the probability law of $H$ (up to a scaling constant $c$ ) it is sufficient to choose one particular such $L$. Choosing $L=N$, i.e. a standard Poisson process, we know from Mai (2018) that $\left\{H_{t}\right\}_{t \geq 0} \stackrel{d}{=}\left\{M_{\theta} t^{1 / \theta}\right\}_{t \geq 0}$ with a $\theta$-stable random variable $M_{\theta}$. In contrast to Lévy subordinators, which have independent increments, this stochastic process looks peculiar at first glimpse. The whole path of the process is already known if one just observes $H_{t}$ for one $t>0$. This phenomenon is studied in more detail in Section 2.3.

2.3. The natural filtration of $H$. We investigate the amount of information one can obtain by observing the process $H$ up to some time $t>0$. We define by $\mathcal{F}_{t}^{H}=\sigma\left(H_{s}: 0 \leq s \leq t\right)$ the information from observing $H$ up to time $t>0$, similarly we define and interpret $\mathcal{F}_{t}^{L}$. We have already seen in Example 2.12 that $\left\{\mathcal{F}_{t}^{H}\right\}_{t \geq 0}$ can be a quite trivial filtration. Here is a second motivating example with the same phenomenon, although significantly more difficult to see.

Example 2.13 (The case $L=N$ and $F(x)=1-\exp (-x)$ ). Let $L=N$ be a Poisson process with unit intensity, whose sequence of consecutive jump times we denote by $\left\{\tau_{k}\right\}_{k \geq 1}$, and $F$ the distribution function of the unit exponential law. In particular, $\mathrm{d} F$ has unbounded support. One can show that $\mathcal{F}_{t}^{H}=\mathcal{F}_{\infty}^{H}$ for all $t>0$. In words, this means that the whole path of $H$ is determined completely by the path on $[0, t]$ for arbitrarily small $t>0$. To see this, we show that the function

$$
z \mapsto H_{z}=\sum_{k \geq 1}-\log \left(1-e^{-\frac{\tau_{k}}{z}}\right)
$$

is almost surely holomorphic on $\mathbb{C}_{+}:=\{z \in \mathbb{C}: \mathcal{R}(z)>0\}$. Since holomorphic functions on $\mathbb{C}_{+}$are determined everywhere, once they are determined on a small real interval, such as $(0, t) \subset \mathbb{C}_{+}$for $t>0$, the claim follows. Why is $z \mapsto H_{z}$ holomorphic? Using Gilman et al. (2007, Theorem 7.2, p. 124), it is sufficient to prove that the defining series of $H_{z}$ converges uniformly on all compact subsets of $\mathbb{C}_{+}$. Each compact subset of $\mathbb{C}_{+}$is contained within a set of the form $\left\{z \in \mathbb{C}_{+}\right.$: $\epsilon \leq|z| \leq c, \mathcal{R}(z) \geq \epsilon\}$ for some constants $\infty>c>\epsilon>0$. On this set, we have for arbitrary real $x>0$ that

$$
\left|e^{-\frac{x}{z}}\right|=e^{-\frac{x \mathcal{R}(z)}{|z|^{2}}} \leq e^{-\frac{x \epsilon}{c^{2}}} .
$$

Furthermore, the law of the iterated logarithm implies that (a.s.)

$$
\liminf _{k \rightarrow \infty} \frac{\tau_{k}-k}{\sqrt{2 k \log (\log (k))}}=-1
$$

Consequently, we obtain for almost all $k \in \mathbb{N}$ the estimate

$$
\tau_{k}=k+\sqrt{2 k \log (\log (k))} \frac{\tau_{k}-k}{\sqrt{2 k \log (\log (k))}} \geq k-\sqrt{2 k \log (\log (k))}(1+\epsilon) \geq k / 2 .
$$


These estimates, together with the identity $-\log (1-x)=\sum_{m \geq 1} x^{m} / m$ for real $x \in[0,1)$, imply for $n$ large enough that

$$
\begin{aligned}
\left|\sum_{k \geq n}-\log \left(1-e^{-\frac{\tau_{k}}{z}}\right)\right| & \leq \sum_{k \geq n} \sum_{m \geq 1} \frac{1}{m}\left|e^{-\frac{\tau_{k} m}{z}}\right| \stackrel{(2.7)}{\leq} \sum_{k \geq n} \sum_{m \geq 1} \frac{1}{m} e^{-\frac{\tau_{k} m \epsilon}{c^{2}}} \\
& \stackrel{(2.8)}{\leq} \sum_{k \geq n} \sum_{m \geq 1} \frac{1}{m} e^{-\frac{k m \epsilon}{2 c^{2}}}=\sum_{m \geq 1} \frac{1}{m} \frac{e^{-\frac{n m \epsilon}{2 c^{2}}}}{1-e^{-\frac{m \epsilon}{2 c^{2}}}} \\
& \leq \frac{1}{1-e^{-\frac{\epsilon}{2 c^{2}}}} \frac{e^{-\frac{n \epsilon}{2 c^{2}}}}{1-e^{-\frac{n \epsilon}{2 c^{2}}}} .
\end{aligned}
$$

Since the last expression tends to zero as $n \rightarrow \infty$, uniform convergence of the defining series on all compact subsets of $\mathbb{C}_{+}$is shown.

The observation in Examples 2.12 and 2.13 is a general rule, as the following lemma shows.

Lemma 2.14 (Filtering out $L$ from $H$ ). Let $(F, L)$ be admissible. We denote by $u_{F}=F^{-1}(1)$ the right-end point of the support of $\mathrm{d} F$. Then $\mathcal{F}_{t}^{H}=\mathcal{F}_{u_{F} t}^{L}$ for arbitrary $t \geq 0$. In particular, if $u_{F}=\infty$ this implies for arbitrary $t>0$ that

$$
\mathcal{F}_{t}^{H}=\mathcal{F}_{\infty}^{L}=\mathcal{F}_{\infty}^{H} .
$$

Proof: We first assume that $u_{F}<\infty$, which implies that the defining integral representation (1.2) of $H$ becomes the series representation

$$
H_{t}=\sum_{k: \tau_{k} \leq u_{F} t}-\log \left(F\left(\frac{\tau_{k}}{t}-\right)\right) J_{k},
$$

where $\tau_{1}<\tau_{2}<\ldots$ denote the at most countably many jump times of $L$ in $\left(0, u_{F} t\right)$ with respective jump sizes $J_{1}, J_{2}, \ldots$.

The inclusion $\mathcal{F}_{t}^{H} \subset \mathcal{F}_{u_{F}}^{L}$ is immediate from (2.9).

The inclusion $\mathcal{F}_{t}^{H} \supset \mathcal{F}_{u_{F}}^{L}$ requires us to recover the jump times $\left\{\tau_{k}\right\}$ and jump sizes $\left\{J_{k}\right\}$ of $L$ up to time $u_{F} t$ from observing the process $H$ up to time $t>0$. This can be done inductively.

If $H_{t}=0$, then $\tau_{1}>u_{F} t$ and we are done. Else, we recover the first jump time by $\tau_{1}:=\inf \left\{s \in[0, t]: H_{s}>0\right\}$ as well as $J_{1}=\lim _{\epsilon}{ } H_{\tau_{1}+\epsilon} /-\log \left(F\left(\frac{\tau_{1}}{\tau_{1}+\epsilon}\right)\right)$.

If $H_{t}=-\log \left(F\left(\frac{\tau_{1}}{t}-\right)\right) J_{1}$, then $\tau_{2}>u_{F} t$ and we are done. Else, we recover the second jump time by $\tau_{2}:=\inf \left\{s \in\left(\tau_{1}, t\right]: H_{s}+\log \left(F\left(\frac{\tau_{1}}{s}-\right)\right) J_{1}>0\right\}$ as well as

$$
J_{2}=\lim _{\epsilon \searrow 0} \frac{H_{\tau_{2}+\epsilon}+\log \left(F\left(\frac{\tau_{1}}{\tau_{2}+\epsilon}\right)\right) J_{1}}{-\log \left(F\left(\frac{\tau_{2}}{\tau_{2}+\epsilon}\right)\right)} .
$$

This procedure is repeated until either $\tau_{n}>u_{F} t$ for some $n \in \mathbb{N}$ if $L$ is of compound Poisson type, or ad infinitum otherwise.

Before we prove the statement in the case $u_{F}=\infty$, we collect some further useful facts in the case $u_{F}<\infty$. We decompose the increments of $H$ into two parts. To 
this end, we observe for $h>0$ using (1.3) that

$$
\begin{aligned}
H_{t+h}-H_{t}=\int_{0}^{u_{F}}\left(L_{s(t+h)}-L_{u_{F} t}+L_{u_{F} t}-L_{s t}\right) \mathrm{d}(\log F(s)) \\
=\int_{\frac{u_{F} t}{t+h}}^{u_{F}}\left(L_{s(t+h)}-L_{u_{F} t}\right) \mathrm{d}(\log F(s))+\int_{0}^{\frac{u_{F} t}{t+h}}\left(L_{s(t+h)}-L_{u_{F} t}\right) \mathrm{d}(\log F(s)) \\
\quad+\int_{0}^{u_{F}}\left(L_{u_{F} t}-L_{s} t\right) \mathrm{d}(\log F(s)) \\
=\underbrace{\int_{u_{F} t}^{u_{F}}(t+h)}_{\text {positive random variable, independent of } \mathcal{F}_{u_{F}}^{L} t}(\underbrace{\int_{0}^{u_{F} t}\left(L_{u_{F} t}-L_{v}\right) \mathrm{d}\left(\log \frac{F(v / t)}{F(v /(t+h))}\right.}_{\text {positive random variable, } \mathcal{F}_{u_{F}}^{L} t^{- \text {measurable }}} \\
=: \text { : } X_{1}+X_{2} .
\end{aligned}
$$

We can continue to investigate $X_{1}$ and $X_{2}$ and find:

$$
\begin{aligned}
& X_{1} \stackrel{d}{=} \int_{0}^{u_{F}} L_{x y} \mathrm{~d}\left(\log F\left(\frac{h y+u_{F} t}{t+h}\right)\right)=-\int_{0}^{u_{F}} \log F\left(\frac{h y+u_{F} t}{h+t}-\right) \mathrm{d} L_{h y} \geq 0 \\
& X_{2}=\int_{0}^{u_{F} t}-\log F\left(\frac{v}{t+h}-\right) \mathrm{d} L_{v}-H_{t} \geq 0 .
\end{aligned}
$$

Now we prove the claim assuming $u_{F}=\infty$. We fix $t, h>0$ and approximate $F \in \mathfrak{F}$ by the sequence of distribution functions $F_{n}(x):=\mathbf{1}_{\{x \geq n\}}+\mathbf{1}_{\{x<n\}} F(x)$, for $n \in \mathbb{N}$, and observe that

$$
H_{t}^{(n)}:=H_{t}^{\left(F_{n}, L\right)}=\int_{0}^{n}-\log F(s / t-) \mathrm{d} L_{s} \longrightarrow H_{t}=H_{t}^{(F, L)} \text { for } n \rightarrow \infty .
$$

This, in turn, implies that $H_{t+h}^{(n)}-H_{t}^{(n)} \longrightarrow H_{t+h}-H_{t}$ for $n \rightarrow \infty$ (almost surely). As shown above, the random variable $H_{t+h}^{(n)}-H_{t}^{(n)}$ equals the sum of $X_{2}^{(n)}$ that is measurable with respect to $\mathcal{F}_{t}^{H^{(n)}}$, and another part $X_{1}^{(n)}$ which is independent thereof. We observe further from (2.10) that

$$
X_{2}^{(n)}=\int_{0}^{\infty}-\log F_{n}\left(\frac{v}{t+h}-\right) \mathbf{1}_{\left\{v<u_{F_{n}} t\right\}} \mathrm{d} L_{v}-H_{t} .
$$

Since $F_{n}$ converges in a monotonically decreasing manner to $F$, the integrand in the last expression converges in a monotonically increasing fashion to $-\log F(v /(t+$ $h)-)$. Thus, the monotone convergence theorem, applied pathwise, shows that $X_{2}^{(n)}$ converges almost surely to $H_{t+h}-H_{t}$. Consequently, $X_{1}^{(n)}$ tends to zero almost surely as $n \rightarrow \infty$. Since $X_{2}^{(n)}$ is measurable with respect to $\mathcal{F}_{t}^{H^{(n)}}$ for all $n$, we conclude that $H_{t+h}-H_{t}$ is measurable with respect to $\mathcal{F}_{t}^{H}$, finishing the argument.

Example 2.15 (The case $-\log F(x)=(1-x)_{+}$). The resulting process $H$ is called Holmgren-Liouville convoluted subordinator in Bender and Marquardt (2009) and has also been studied in Bernhart et al. (2015). Investing it in the present context (note that $u_{F}=1$ ), we find for $X_{1}, X_{2}$ defined as in the proof of Lemma 2.14 above 
that

$$
\begin{aligned}
\Psi_{1}(\alpha) & :=-\log \left(\mathbb{E}\left[e^{-\alpha X_{1}}\right]\right)=h \int_{0}^{1} \Psi_{L}\left(\alpha\left(1-\frac{h y+t}{h+t}\right)\right) \mathrm{d} y \\
& =h \int_{0}^{1} \Psi_{L}\left(\alpha(1-y) \frac{h}{h+t}\right) \mathrm{d} y=h \Psi_{H}\left(\alpha \frac{h}{h+t}\right), \\
X_{2} & =\frac{h}{h+t}\left(L_{t}-H_{t}\right) .
\end{aligned}
$$

The function $\Psi_{1}$ tends to zero as $t$ increases to infinity, provided $\Psi_{L}$ has no killing. This is intuitive in the sense that the larger $t$, the more we know about the increment $H_{t+h}-H_{t}$ for fixed $h>0$.

Example 2.16 (The case $F(x)=\exp \left(-c_{\theta} x^{-1 / \theta}\right)$ ). Let $M_{\theta}$ be a positive random variable with Laplace transform $x \mapsto \exp \left(-x^{\theta}\right), \theta \in(0,1)$, then the associated strong IDT process is given as $H_{t}=M_{\theta} t^{1 / \theta}$, see Example 2.12. This is a peculiar stochastic process, as observing it at only one fixed $t>0$ corresponds to knowing it everywhere. We have furthermore seen in Example 2.12 that the choice of the Lévy subordinator $L$ is arbitrary, provided admissibility. In particular, we are free to choose a compound Poisson process with unit intensity and unit exponentially distributed jumps, i.e. $\Psi_{L}(x)=x /(x+1)$, which is a convenient choice for the following considerations. We truncate $F$ via $F_{n}(x):=\mathbf{1}_{\{x \geq n\}}+\mathbf{1}_{\{x<n\}} F(x)$ like in the proof of Lemma 2.14. Clearly, we then have $u_{F_{n}}=n<\infty$ and $F_{n}$ converges to $F$ pointwise. We find for $X_{1}^{(n)}, X_{2}^{(n)}$ as defined in the proof of Lemma 2.14 that

$$
\begin{aligned}
& -\log \left(\mathbb{E}\left[e^{-\alpha X_{1}^{(n)}}\right]\right)=h \int_{0}^{n} \frac{c_{\theta}(h+t)^{1 / \theta}}{c_{\theta}(h+t)^{1 / \theta}+(h y+n t)^{1 / \theta}} \mathrm{d} y \longrightarrow 0, \quad(n \rightarrow \infty), \\
& X_{2}^{(n)}=c_{\theta}\left((t+h)^{1 / \theta}-t^{1 / \theta}\right) \int_{0}^{n t} v^{-1 / \theta} \mathrm{d} L_{v} \longrightarrow H_{t+h}-H_{t}, \quad(n \rightarrow \infty),
\end{aligned}
$$

both observations confirming our knowledge about $H_{t}$ from Lemma 2.14.

\section{Application 1: Simulation of extreme-value copulas}

Throughout this section, we fix one admissible pair $(F, L)$. The fact that $\ell$ in Lemma 2.3 is homogeneous of order 1 implies by virtue of Mai and Scherer (2014, Theorem 5.3) that the infinite exchangeable sequence of random variables

$$
Y_{k}:=\inf \left\{t>0: H_{t}>\epsilon_{k}\right\}, \quad k \in \mathbb{N},
$$

with $\left\{\epsilon_{k}\right\}_{k \in \mathbb{N}}$ independent unit exponentials, independent of $H$, is min-stable multivariate exponential with survival function given by

$$
\mathbb{P}\left(Y_{1}>t_{1}, Y_{2}>t_{2}, \ldots\right)=e^{-\ell(\vec{t})}, \quad \vec{t} \in[0, \infty)_{00}^{\mathbb{N}} .
$$

This means that $\min \left\{Y_{1} / t_{1}, Y_{2} / t_{2}, \ldots\right\}$ has a univariate exponential distribution with rate $\ell(\vec{t})$, not all $t_{k}$ equal to zero. The exponential rate of each component $Y_{k}$ equals $\Psi_{H}(1)$ and it is convenient to normalize it to $\Psi_{H}(1)=1$, which then implies that the $d$-variate function $\ell_{d}\left(t_{1}, \ldots, t_{d}\right):=\ell\left(t_{1}, \ldots, t_{d}, 0,0, \ldots\right)$ defines a so-called stable tail dependence function in dimension $d$. If for $(F, L)$ the law of $H=H^{(F, L)}$ does not satisfy $\Psi_{H}(1)=1$, we can always change from $F$ to $F(c$.) for some $c>0$ such that $H^{(F(c .), L)}$ has this property. This $c$ is even unique, i.e. for any given $(F, L)$ there is a unique $c>0$ such that $H^{(F(c .), L)}$ satisfies $\Psi_{H}(1)=1$. Given this 
normalization, the function $C_{d}\left(u_{1}, \ldots, u_{d}\right):=\exp \left\{-\ell_{d}\left(-\log \left(u_{1}\right), \ldots,-\log \left(u_{d}\right)\right)\right\}$, $u_{1}, \ldots, u_{d} \in(0,1)$, defines a so-called extreme-value copula. For background on the latter, the reader is referred to Joe (1997); Nelsen (2006); Gudendorf and Segers (2010). Loosely speaking, extreme-value copulas are the dependence structures behind the limit of appropriately normalized componentwise maxima of independent and identically distributed random vectors, which is of paramount interest in multivariate extreme-value theory. The relationship between strong IDT processes and multivariate extreme-value theory has already been investigated in the present authors' references Mai and Scherer (2014); Bernhart et al. (2015); Mai (2018, 2019). On one hand, the stochastic model (3.1) can directly be used to simulate the random vector $\mathbf{U}:=\exp (-\mathbf{Y}) \sim C_{d}$, where $\mathbf{Y}=\left(Y_{1}, \ldots, Y_{d}\right)$. However, due to the in general inconvenient integral definition of $H$ in (1.2), the infinite series representation of $H$ in Lemma 2.7, and the fact that the increments of $H$ are typically not independent, this is a non-trivial task in general, although feasible in particular cases, an example with $L$ being a Poisson process and support of $\mathrm{d} F$ bounded is provided in Mai (2018, Section 3.1). However, there is an alternative approach to accomplish the simulation in the general case, as described in the remainder of this section.

It is well-known from de Haan and Resnick (1977); Ressel (2013) that the stable tail-dependence function $\ell_{d}$ is uniquely associated with a probability measure on the unit simplex $S_{d}:=\left\{\left(q_{1}, \ldots, q_{d}\right) \in[0,1]^{d}: q_{1}+\ldots+q_{d}=1\right\}$ subject to the constraint that each component has expected value $1 / d$. To wit, there exists a random vector $\mathbf{Q}=\left(Q_{1}, \ldots, Q_{d}\right)$, uniquely determined in law, taking values in $S_{d}$ and satisfying $\mathbb{E}\left[Q_{k}\right]=1 / d$ for each $k=1, \ldots, d$, such that

$$
\ell_{d}\left(t_{1}, \ldots, t_{d}\right)=d \mathbb{E}\left[\max \left\{t_{1} Q_{1}, \ldots, t_{d} Q_{d}\right\}\right], \quad t_{1}, \ldots, t_{d} \geq 0
$$

which is called the Pickands representation of $\ell_{d}$, named after Pickands III (1981). It is important to notice that $\mathbf{Q}=\mathbf{Q}^{[d]}$ depends on the dimension $d$. In particular, in our situation where $d$ is arbitrary the first $d$ components of $\mathbf{Q}^{[d+1]}$ are not equal in distribution to $\mathbf{Q}^{[d]}$, not even when re-scaled. In order to simplify notation, however, we omit to highlight this dependence on $d$ for the rest of this section.

The simulation algorithm in Dombry et al. (2016, Algorithm 1), based on a seminal idea by Schlather (2002), shows how to simulate a random vector $\mathbf{U}=$ $\exp (-\mathbf{Y}) \sim C_{d}$ exactly and efficiently, if one has at hand a simulation algorithm for the vector $\mathbf{Q}$. More precisely, it is shown that

$$
\begin{gathered}
\mathbf{Y} \stackrel{d}{=}\left(\frac{1}{Z_{1}^{(K)}}, \ldots, \frac{1}{Z_{d}^{(K)}}\right) \\
\left(Z_{1}^{(n)}, \ldots, Z_{d}^{(n)}\right) \stackrel{d}{=}\left(\max _{k=1, \ldots, n}\left\{\frac{d Q_{1}^{(k)}}{\epsilon_{1}+\ldots+\epsilon_{k}}\right\}, \ldots, \max _{k=1, \ldots, n}\left\{\frac{d Q_{d}^{(k)}}{\epsilon_{1}+\ldots+\epsilon_{k}}\right\}\right), n \geq 1
\end{gathered}
$$

where $\left\{\mathbf{Q}^{(k)}\right\}_{k \geq 1}$ denote independent copies of $\mathbf{Q}$, independently of $\left\{\epsilon_{k}\right\}_{k \geq 1}$ iid unit exponentials, and $K$ equals the smallest $n \in \mathbb{N}$ for which $d /\left(\epsilon_{1}+\ldots+\epsilon_{n+1}\right)$ is smaller than the minimal component of $\left(Z_{1}^{(n)}, \ldots, Z_{d}^{(n)}\right)$. Thus, deriving an efficient and exact simulation algorithm for $\mathbf{Q}$ is essentially the key to deriving an efficient and exact simulation algorithm for the extreme-value copula $C_{d}$ along the model (3.3). The purpose of the present section is to demonstrate how this is possible. Concluding, one concrete application of the stochastic processes considered in the 
present article is to enlarge the repertoire of extreme-value copulas for which exact and efficient simulation strategies are available.

3.1. The Pickands representation of $\ell_{d}$. We assume that $\Psi_{H}(1)=1$ so that $\ell_{d}$ is a proper stable tail dependence function. This condition implies that

$$
\int_{(0, \infty]} \Psi_{F}(z) \nu_{L}(\mathrm{~d} z)=1
$$

so that $\Psi_{F}(z) \nu_{L}(\mathrm{~d} z)$ is a probability measure on $(0, \infty]$. Notice that this probability measure has already been important in Lemma 2.7. In the sequel, we show that it also occupies a commanding role when determining the Pickands representation of $\ell_{d}$. Denoting by $Z$ a generic random variable drawn from this probability law, observe that

$$
\begin{aligned}
& \ell_{d}\left(t_{1}, \ldots, t_{d}\right)=\int_{(0, \infty]} \iint_{[0, \infty)^{d}} \max \left\{t_{1} x_{1}, \ldots, t_{d} x_{d}\right\} \prod_{i=1}^{d} \mathrm{~d} F^{y}\left(x_{i}\right) \nu_{L}(\mathrm{~d} y) \\
& =\mathbb{E}\left[\frac{1}{\Psi_{F}(Z)} \iint_{[0, \infty)^{d}} \max \left\{t_{1} x_{1}, \ldots, t_{d} x_{d}\right\} \prod_{i=1}^{d} \mathrm{~d} F^{Z}\left(x_{i}\right)\right] \\
& =d \mathbb{E}\left[\iint_{[0, \infty)^{d}} \max _{i=1, \ldots, d}\left\{t_{i} \frac{x_{i}}{\sum_{k=1}^{d} x_{k}}\right\} \frac{1}{d} \sum_{k=1}^{d} \frac{x_{k} \mathrm{~d} F^{Z}\left(x_{k}\right)}{\Psi_{F}(Z)} \prod_{i \neq k}^{d} \mathrm{~d} F^{Z}\left(x_{k}\right)\right] .
\end{aligned}
$$

The important observation from this computation is that the vector

$$
\left(q_{1}, \ldots, q_{d}\right):=\left(\frac{x_{1}}{\sum_{k=1}^{d} x_{k}}, \ldots, \frac{x_{d}}{\sum_{k=1}^{d} x_{k}}\right)
$$

takes values in $S_{d}$ and, conditioned on $Z$, the measure

$$
\frac{1}{d} \sum_{k=1}^{d} \frac{x_{k} \mathrm{~d} F^{Z}\left(x_{k}\right)}{\Psi_{F}(Z)} \prod_{i \neq k}^{d} \mathrm{~d} F^{Z}\left(x_{k}\right)
$$

is a probability measure on $[0, \infty)^{d}$. To see this, notice that conditioned on $Z$, the measure $\frac{x \mathrm{~d} F^{Z}(x)}{\Psi_{F}(Z)}$ is a probability measure on $[0, \infty)$, since

$$
\int_{[0, \infty)} \frac{x \mathrm{~d} F^{Z}(x)}{\Psi_{F}(Z)}=\frac{\int_{0}^{\infty} 1-F(s)^{Z} \mathrm{~d} s}{\Psi_{F}(Z)}=1 .
$$

Consequently, we have found the unique Pickands dependence measure, as summarized in the following lemma.

Lemma 3.1 (Pickands representation of $\ell_{d}$ ). A random sample of $\boldsymbol{Q}$, which is uniquely determined in law by (3.2), can be drawn according to the following algorithm:

- Draw D uniformly distributed on $\{1, \ldots, d\}$.

- Draw a sample of the random variable $Z \sim \Psi_{F}(z) \nu_{L}(\mathrm{~d} z)$.

- Draw d independent and identically distributed random variables $X_{1}, \ldots, X_{d}$ from the distribution function $F^{Z}$.

- Draw a random variable $M$ from the probability law $x \mathrm{~d} F^{Z}(x) / \Psi_{F}(Z)$. 
- Compute the random vector $\left(W_{1}, \ldots, W_{d}\right)$, defined by

$$
W_{k}:=\left\{\begin{array}{ll}
X_{k} & , \text { if } k=D \\
M & , \text { else }
\end{array} .\right.
$$

- Return

$$
\boldsymbol{Q}:=\left(\frac{W_{1}}{\sum_{k=1}^{d} W_{k}}, \ldots, \frac{W_{d}}{\sum_{k=1}^{d} W_{k}}\right) .
$$

Remark 3.2 (Expected runtime of the algorithm in Lemma 3.1). When using the stochastic representation (3.3) together with Lemma 3.1 to simulate the extremevalue copula $C_{d}$, the runtime of the simulation algorithm is random itself. However, Dombry et al. (2016, Proposition 4) shows that the expected value of $K$ in (3.3) equals $d \mathbb{E}\left[\max \left\{Y_{1}, \ldots, Y_{d}\right\}\right]$, which in the present situation can be computed in closed form by

$$
\begin{aligned}
\mathbb{E}[K] & =d \mathbb{E}\left[\max \left\{Y_{1}, \ldots, Y_{d}\right\}\right]=d \mathbb{E}\left[\int_{0}^{\infty}\left(1-\left(1-e^{-H_{t}}\right)^{d}\right) \mathrm{d} t\right] \\
& =-d \sum_{k=1}^{d}\left(\begin{array}{l}
d \\
k
\end{array}\right)(-1)^{k} \int_{0}^{\infty} \mathbb{E}\left[e^{-k H_{t}}\right] \mathrm{d} t=-d \sum_{k=1}^{d}\left(\begin{array}{l}
d \\
k
\end{array}\right)(-1)^{k} \int_{0}^{\infty} e^{-t \Psi_{H}(k)} \mathrm{d} t \\
& =-d \sum_{k=1}^{d}\left(\begin{array}{l}
d \\
k
\end{array}\right) \frac{(-1)^{k}}{\Psi_{H}(k)} \leq d^{2} .
\end{aligned}
$$

The last estimate follows from the estimate

$$
\mathbb{E}\left[\int_{0}^{\infty}\left(1-\left(1-e^{-H_{t}}\right)^{d}\right) \mathrm{d} t\right]=\mathbb{E}\left[\Psi_{1-e^{-H}}(d)\right] \leq \underbrace{\mathbb{E}\left[\Psi_{1-e^{-H}}(1)\right]}_{=1 \text { by normalization }} d,
$$

where the inequality follows from the argument in the proof of Lemma 2.4. Since the simulation of $\mathbf{Q}$ in Lemma 3.1 itself is apparently of linear order in the dimension $d$, the total expected runtime for the exact simulation of the extreme-value copula $C_{d}$ according to the representation (3.3) with the help of Lemma 3.1 has expected order between $d^{2}$ and $d^{3}$ and can be computed explicitly in terms of the Bernstein function $\Psi_{H}$.

In the sequel, we work out some concrete examples, demonstrating the versatility of Lemma 3.1.

\subsection{Examples.}

Example 3.3 (The Lévy subordinator case). Consider the distribution function $F(x)=\exp (-1)+(1-\exp (-1)) \mathbf{1}_{[1, \infty)}(x)$ with associated Bernstein function

$$
\Psi_{F}(x)=1-e^{-x}, \quad x \geq 0 .
$$

An arbitrary Lévy subordinator $L$ leads to an admissible pair $(F, L)$ and obviously $H=L$. Furthermore, $\Psi_{H}(1)=1$ is satisfied whenever $\Psi_{L}(1)=1$. It is wellknown that $C_{d}$ equals the survival copula of a so-called Marshall-Olkin distribution, named after Marshall and Olkin (1967), for details see Mai and Scherer (2009, 2011). Furthermore, it is observed for $z>0$ that a random variable $X \sim F^{z}$ is Bernoulli-distributed with success probability $1-\exp (-z)$. In order to simulate from the Pickands measure, additionally required is also a simulation algorithm for 
$M \sim x \mathrm{~d} F^{z}(x) / \Psi_{F}(z)$ with given $z$. With $X_{z}$ a Bernoulli random variable with success probability $1-\exp (-z)$, it is observed that

$$
\mathbb{P}(M \leq x)=\int_{0}^{x} \frac{s}{\Psi_{F}(z)} \mathrm{d} F^{z}(s)=\frac{1}{1-e^{-z}} \mathbb{E}\left[\mathbf{1}_{\left\{X_{z} \leq x\right\}} X_{z}\right]=\mathbf{1}_{\{x \geq 1\}},
$$

hence $M \equiv 1$. Summarizing, the random vector $\mathbf{Q}=\left(Q_{1}, \ldots, Q_{d}\right)$ can be simulated as follows:

- Draw a random variable $D$ which is uniformly distributed on $\{1, \ldots, d\}$.

- Draw the random variable $Z \sim(1-\exp (-z)) \nu_{L}(\mathrm{~d} z)$.

- Simulate $X_{1}, \ldots, X_{d}$ iid Bernoulli variables with success probability 1 $\exp (-Z)$.

- Compute the random vector $\left(W_{1}, \ldots, W_{d}\right)$ as

$$
W_{k}:=\left\{\begin{array}{ll}
X_{k} & , \text { if } k \neq D \\
1 & , \text { if } k=D
\end{array} .\right.
$$

- Return $\mathbf{Q}$, where $Q_{k}:=W_{k} /\left(\sum_{i=1}^{d} W_{i}\right)$, for $k=1, \ldots, d$.

To illustrate, we consider one particular Lévy subordinator, which is of interest in the context of regenerative composition structures, see Gnedin and Pitman (2005, Section 8). Define the Lévy measure by

$$
\nu_{L}(\mathrm{~d} x)=\frac{e^{-\theta x}}{\left(1-e^{-x}\right)^{\alpha+1}}\left\{\alpha e^{-x}+\theta\left(1-e^{-x}\right)\right\} \frac{\Gamma(2+\theta-\alpha)}{\Gamma(\theta+1) \Gamma(1-\alpha)},
$$

with $\theta \geq 0$ and $0 \leq \alpha<1$. Then

$$
\Psi_{L}(x)=x \frac{\Gamma(x+\theta) \Gamma(2+\theta-\alpha)}{\Gamma(1+\theta) \Gamma(x+\theta+1-\alpha)}
$$

and $\Psi_{L}(1)=1$. The required probability distribution of $Z$ has density

$$
\begin{aligned}
z & \mapsto e^{-z \theta}\left(1-e^{-z}\right)^{-\alpha}\left\{\alpha e^{-z}+\theta\left(1-e^{-z}\right)\right\} \frac{\Gamma(2-\alpha+\theta)}{\Gamma(\theta+1) \Gamma(1-\alpha)} \\
& =(1-\alpha) g_{\theta, 2-\alpha}(z)+\alpha g_{\theta+1,1-\alpha}(z),
\end{aligned}
$$

where $g_{p, q}$ denotes the density of $-\log (B)$ for $B$ having a Beta distribution with parameters $(p, q)$. Apparently, this mixture of transformed Beta distributions can be simulated exactly and efficiently, making the simulation algorithm for $\left(Y_{1}, \ldots, Y_{d}\right)$ feasible. Notice that this simulation of $\left(Y_{1}, \ldots, Y_{d}\right)$ implies an efficient alternative simulation algorithm for the random variable $\mathcal{C}_{d}$ in Gnedin and Pitman (2005, Section 8), as $\mathcal{C}_{d}$ is defined as the composition of the integer $d$ obtained as the vector of cardinalities of the subsets of $\left\{Y_{1}, \ldots, Y_{d}\right\}$ with identical elements.

Example 3.4 (The case $L=N$ ). In the special case when $L=N$ is a standard Poisson process, one observes that $Z \equiv 1$ and Lemma 3.1 boils down to a result in Mai (2018, Lemma 4). It can thus be viewed as a generalization thereof.

Example 3.5 (The case $F(x)=\min \{x, 1\}$ ). The stable tail dependence function $\ell_{d}$ is studied in Bernhart et al. (2015, Theorem 2). However, no simulation algorithm for $\mathbf{Q}$ has been found in that reference, a gap which we now fill. It is observed that

$$
\Psi_{F}(y)=\int_{0}^{1}\left(1-s^{y}\right) \mathrm{d} s=\frac{y}{y+1}, \quad y \geq 0,
$$


is the Bernstein function associated with a compound Poisson subordinator with unit exponential jumps and unit intensity. Hence, any $L$ leads to an admissible pair $(F, L)$ by Lemma 2.6. In order to ensure $\Psi_{H}(1)=1, \nu_{L}$ must be normalized such that $\int_{(0, \infty]} z /(z+1) \nu_{L}(\mathrm{~d} z)=1$. For any fixed $z>0$ the distribution function $F(x)^{z}=\min \left\{x^{z}, 1\right\}$ is trivial to simulate from via the inversion method, see Mai and Scherer (2017, p. 234). Furthermore, the distribution function of the random variable $M \sim x \mathrm{~d} F^{z}(x) / \Psi_{F}(z)$ is given by $x \mapsto \min \left\{x^{z+1}, 1\right\}$, which is also easy to simulate from by the inversion method. Consequently, the simulation algorithm in Lemma 3.1 is straightforward to implement, whenever the Lévy subordinator $L$ is chosen such that the probability law of $Z$, that is

$$
\mathbb{P}(Z \in \mathrm{d} z)=\frac{z}{z+1} \nu_{L}(\mathrm{~d} z)
$$

can be simulated from.

Example 3.6 (The case $F(x)=\min \{\exp (x-1), 1\}$ ). Recall from Example 2.15 that the resulting process $H$ is the Holmgren-Liouville convoluted subordinator considered in Bender and Marquardt (2009). The stable tail dependence function $\ell_{d}$ is computed in closed form in Bernhart et al. (2015, Theorem 1). It is given by

$$
\begin{aligned}
& \ell_{d}\left(x_{1}, \ldots, x_{d}\right) \\
& =\frac{d \Psi_{H}(d)}{\sum_{j=1}^{d} x_{[j]}^{-1}}-\sum_{i=1}^{d-1}\left(\frac{d-i+1}{\sum_{j=i}^{d} x_{[j]}^{-1}}-\frac{d-i}{\sum_{j=i+1}^{d} x_{[j]}^{-1}}\right) \Psi_{H}\left(d-i-\sum_{j=i+1}^{d} \frac{x_{[i]}}{x_{[j]}}\right),
\end{aligned}
$$

where $x_{[1]} \leq \ldots \leq x_{[d]}$ is the ordered list of $x_{1}, \ldots, x_{d}$. However, no simulation algorithm for $\mathbf{Q}$ has been found in that reference, a gap which we now fill. It is observed that

$$
\Psi_{F}(y)=\int_{0}^{1}\left(1-e^{y s-y}\right) \mathrm{d} s=1-\frac{1-e^{-y}}{y}, \quad y \geq 0,
$$

is the Bernstein function associated with a compound Poisson subordinator with unit intensity and jumps that are uniformly distributed on $[0,1]$. Hence, any $L$ leads to an admissible pair $(F, L)$ by Lemma 2.6 and Bernhart et al. (2015, Lemma 3) shows that the Bernstein function $\Psi_{H}$ runs through all Bernstein functions with non-increasing Lévy density when $L$ is varied. In order to ensure $\Psi_{H}(1)=1, \nu_{L}$ must be normalized such that $\int_{(0, \infty]}\left(1-\left(1-e^{-z}\right) / z\right) \nu_{L}(\mathrm{~d} z)=1$. For any fixed $z>0$ the distribution function $F(x)^{z}=\min \{\exp (z x-z), 1\}$ is easy to simulate from via the inversion method. Furthermore, the density $f_{M}$ of the random variable $M \sim x \mathrm{~d} F^{z}(x) / \Psi_{F}(z)$ is computed to be

$$
f_{M}(x)=\frac{z^{2} e^{-z}}{z+e^{-z}-1} x e^{x z} \mathbf{1}_{(0,1)}(x) .
$$

This density is bounded and rejection-acceptance sampling with comparison density $g(x)=2 x 1_{(0,1)}(x)$, which itself is easy to simulate via the inversion method, can be implemented to achieve an exact simulation scheme of $M$, see Mai and Scherer (2017, p. 235). Consequently, the simulation algorithm in Lemma 3.1 is straightforward to implement, whenever the Lévy subordinator $L$ is chosen such that the probability law of $Z$, that is

$$
\mathbb{P}(Z \in \mathrm{d} z)=\left(1-\frac{1-e^{-z}}{z}\right) \nu_{L}(\mathrm{~d} z)
$$


can be simulated from.

Figure 3.1 schematically visualizes those admissible pairs $(F, L)$ for which either former literature or the present article provides knowledge about the associated extreme-value copula $C_{d}$, or about the probability distribution of $H_{1}$.

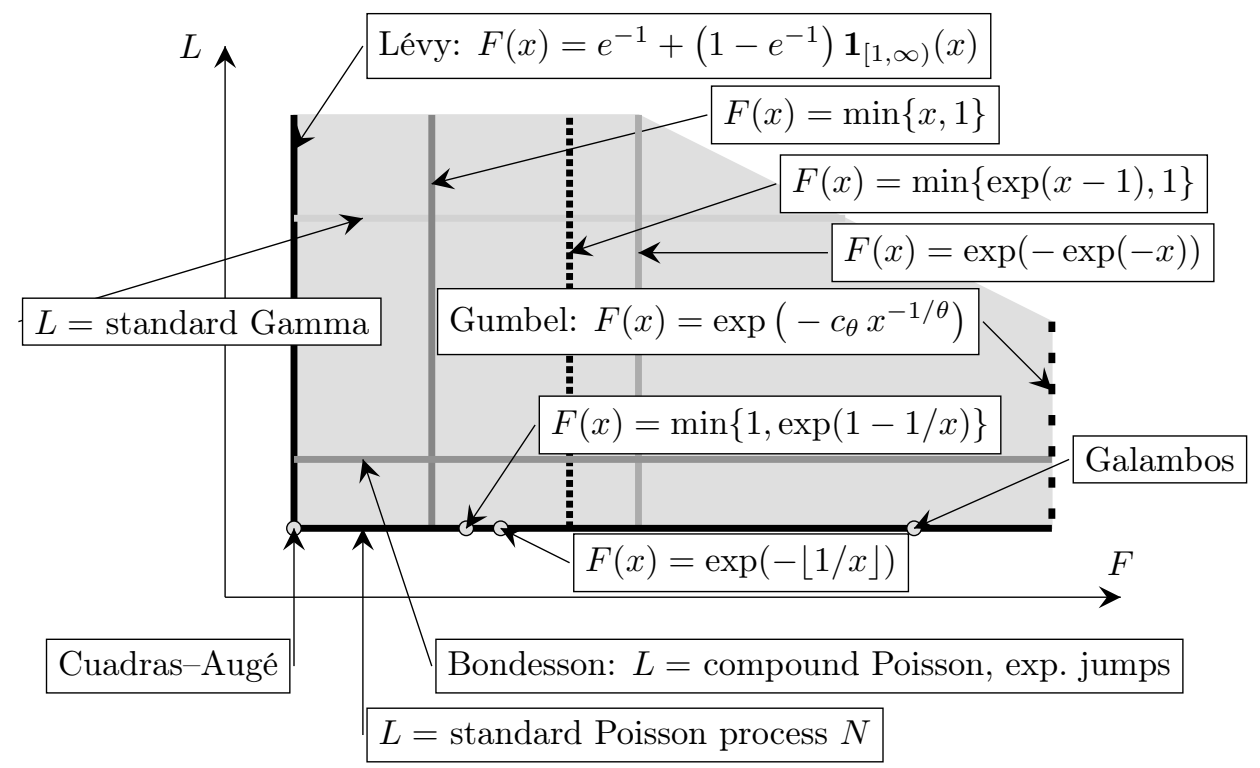

FiguRE 3.1. Illustration of pairs $(F, L)$ occurring in previous literature or the present article. Vertical and horizontal lines of the same greyscale and line-type combination are complementary in the sense of the distributional equality (4.2). The Lévy case is covered in Example 3.3 and the link to Marshall-Olkin copulas originates from Mai and Scherer $(2009,2011)$. The case $F(x)=\min \{x, 1\}$ relates to Example 3.5, see Bernhart et al. (2015) for details. The law of $H_{1}$ in case $F(x)=\exp (-\exp (-x))$ is studied in Behme (2015, Proposition 3.5). The Holmgren-Liouville convoluted subordinator in the case $F(x)=\min \{\exp (x-1), 1\}$ relates to Example 3.6 and has been studied in Bender and Marquardt (2009); James and Zhang (2011); Bernhart et al. (2015). The Gumbel case is covered in Examples 2.12 and 2.16, see also Mai (2018), the name stems from Gumbel $(1960,1961)$. The Galambos and the Cuadras-Augé copulas are named after Galambos (1975) and Cuadras and Augé (1981), respectively. The case of a standard Poisson process is discussed in Example 3.4 and Mai (2018). The cases $F(x)=\exp (-\lfloor 1 / x\rfloor)$ or $F(x)=\min \{1, \exp (1-1 / x)\}$ for $L$ a standard Poisson process appear in Kopp and Molchanov (2018, Example 5.1) or Kopp and Molchanov (2018, Example 4.6), respectively. The Bondesson family relates to Lemma 4.2. The probability law of $H_{1}$ when $L$ is a standard Gamma process is related to generalized Gamma convolutions and Dirichlet means, see James et al. (2008). 


\section{Application 2: Series representations for infinitely divisible laws}

In this section we fix $t=1$ and investigate the probability law of the random variable $H_{1}$, which is an element in $\operatorname{ID}[0, \infty]$. In particular, we are interested in series representations for $H_{1}$ that are suitable for efficient random number generation. We recall from Lemma 2.3 that the Laplace exponent of $H_{1}$ equals

$$
\Psi_{H}(x)=\int_{(0, \infty]} \int_{(0, \infty]}\left(1-e^{-x s u}\right) \nu_{F}(\mathrm{~d} s) \nu_{L}(\mathrm{~d} u), \quad x \geq 0 .
$$

This double integral representation shows that the roles of $F$ and $L$ can be switched without changing the one-dimensional marginal distribution of $H_{1}$. More precisely,

$$
\int_{0}^{\infty}-\log (F(s-)) \mathrm{d} L_{s} \stackrel{d}{=} \int_{0}^{\infty}-\log \left(F^{(L)}(s-)\right) \mathrm{d} L_{s}^{(F)},
$$

where for a given Lévy subordinator $L$ the function $F^{(L)} \in \mathfrak{F}$ is uniquely determined by the equality $\Psi_{F^{(L)}}=\Psi_{L}$, and for a given $F \in \mathfrak{F}$ the Lévy subordinator $L^{(F)}$ is uniquely determined in law by the equality $\Psi_{L^{(F)}}=\Psi_{F}$. Recall that the bijection between the set of Lévy measures on $(0, \infty]$ and $\mathfrak{F}$ is explicitly stated in Lemma 2.2.

The integral definition of $H_{1}$ becomes an infinite series whenever the integrator $L$ is a (driftless) compound Poisson process, i.e. if $\Psi_{L}$ is bounded. Switching the roles of $L$ and $F$ according to the duality (4.2), we also obtain a series representation for $H_{1}$ if $L^{(F)}$ is a (driftless) compound Poisson subordinator, i.e. if $\Psi_{F}$ is bounded.

In the present section we are particularly interested in the case when $L$ is a compound Poisson subordinator with exponentially distributed jumps. However, before we consider this case, we find it educational to briefly point out two choices for $L$ that imply a connection of the current article to previous literature. On the one hand, if $L$ is a standard Gamma process, i.e. $\Psi_{L}(x)=\log (1+x)$, the law of $H_{1}$ runs through the Thorin class when $F$ is varied, see James et al. (2008, Propositions 1.1 and 1.6). On the other hand, the upcoming example is due to Bondesson (1982) and relates to the choice of a standard Poisson process for $L$.

Example 4.1 (Series representations for ID $[0, \infty]$ from duality). On the left-hand side of $(4.2)$ let $F(x)=\exp (-1)+(1-\exp (-1)) \mathbf{1}_{[1, \infty)}(x)$, such that $\Psi_{F}(x)=$ $1-\exp (-x)$ and $L^{(F)}=N$ is a Poisson process with unit intensity, whose unit exponential inter-arrival times we denote by $\left\{\epsilon_{k}\right\}_{k \geq 1}$ and the associated jump times are $\left\{\tau_{k}\right\}_{k \geq 1}$. Then (4.2) becomes

$$
L_{1}=\int_{0}^{\infty}-\log (F(s-)) \mathrm{d} L_{s} \stackrel{d}{=} \sum_{k \geq 1} S_{\nu_{L}}^{-1}(\underbrace{\epsilon_{1}+\ldots+\epsilon_{k}}_{\tau_{k}}) \mathbf{1}_{\{\underbrace{\epsilon_{1}+\ldots+\epsilon_{k}}_{\tau_{k}} \leq \nu_{L}((0, \infty])\}},
$$

where $S_{\nu_{L}}(t):=\nu_{L}((t, \infty])$ is the survival function of the Lévy measure $\nu_{L}$ of $L$, and $S_{\nu_{L}}^{-1}$ its generalized inverse. In particular, this series is almost surely finite in case of a compound Poisson distribution, i.e. if $\Psi_{L}$ is bounded (resp. $\nu_{L}$ is finite). This is more or less the representation of an infinitely divisible law in terms of a series representation involving only independent exponentials that Bondesson (1982) proposes as a basis for his simulation ansatz (restricted to laws on $[0, \infty]$ in the present context). It is of particular use in those cases where the survival function of the Lévy measure has an inverse $S_{\nu_{L}}^{-1}$ in closed form. If, in addition, the Lévy measure is finite, i.e. one has a compound Poisson distribution, one obtains an exact simulation algorithm. When simulating this compound Poisson law, the 
representation is useful if we have no simulation algorithm for the jump size distribution at hand, but we are able to compute the inverse of the Laplace transform of the jump size distribution in closed form.

The series representation in Example 4.1 was based on the choice $L=N$ of a Poisson process as integrator. In the sequel, we choose as integrator a compound Poisson process with unit exponential jumps. In this case, Lemma 4.2 shows that the law of $H_{1}$ lies in the so-called Bondesson family $\mathrm{BO}(0, \infty)$, see Bondesson (1981). This means that the survival function of the Lévy measure equals the Laplace transform of a measure $\rho$ on $(0, \infty)$, called the Stieltjes measure. From an analytical viewpoint, the Bernstein function $\Psi_{H}$, which is said to be complete in this case, can be represented as

$$
\Psi_{H}(x)=\int_{0}^{\infty} \frac{x}{x+u} \rho(\mathrm{d} u), \quad x \geq 0
$$

with the Stieltjes measure $\rho$ on $(0, \infty)$ satisfying $\int_{0}^{\infty}(1+u)^{-1} \rho(\mathrm{d} u)<\infty$. There are examples for laws in $\operatorname{BO}(0, \infty)$ for which the Stieltjes measure $\rho$ is way more convenient to handle than the associated Lévy measure ${ }^{2}$, we provide some examples below. For these cases, Lemma 4.2 provides a series representation in a similar spirit as that for ID $[0, \infty]$ in Example 4.1 .

We fix $L$ as a compound Poisson subordinator with unit exponential jumps and unit intensity, i.e. $\Psi_{L}(x)=x /(x+1)$. Furthermore, we let $F \in \mathfrak{F}$ be arbitrary, and only assume that the left-end point of the support of $X \sim F$ equals zero, which by Lemma 2.2 is equivalent to postulating $\nu_{F}(\{\infty\})=0$, i.e. $\Psi_{F}$ has no killing. In this situation, the measure

$$
\rho_{F}(A):=\nu_{F}\left(\left\{\frac{1}{u}: u \in A\right\}\right)
$$

is well-defined for all Borel sets $A \subset(0, \infty)$, and we observe that

$$
\int_{0}^{\infty} \frac{1}{1+t} \rho_{F}(\mathrm{~d} t)=\int_{0}^{\infty} \frac{u}{1+u} \nu_{F}(\mathrm{~d} u) \leq \int_{0}^{\infty} \min \{u, 1\} \nu_{F}(\mathrm{~d} u)<\infty,
$$

so $\rho_{F}$ is a proper Stieltjes measure. To simplify notation, we denote

$$
\hat{\mathfrak{F}}:=\left\{F \in \mathfrak{F}: \nu_{F}(\{\infty\})=0\right\}
$$

and obtain the following result. The series representation in part (c) is a special case of the series representation discussed in Bondesson (1982, p. 862).

Lemma 4.2 (Series representation in terms of the Stieltjes measure). Let $F \in \hat{\mathfrak{F}}$ and $L$ a compound Poisson subordinator with unit intensity and unit exponentially distributed jumps, i.e. $\Psi_{L}(x)=x /(x+1)$.

(a) The law of $H_{1}$ is in $B O(0, \infty) \subset I D[0, \infty]$ with associated Stieltjes measure $\rho_{F}$.

(b) The mapping $F \mapsto \rho_{F}$, defined by (4.3) and Lemma 2.2, defines a bijection between $\hat{\mathfrak{F}}$ and $B O(0, \infty)$. The inverse mapping $\rho \mapsto F_{\rho}$ is

$$
F_{\rho}(x)=\left\{\begin{array}{ll}
e^{-g_{\rho}^{-1}(x)} & , \text { if } x<\rho((0, \infty)) \\
1 & , \text { else }
\end{array}, \quad g_{\rho}(x):=\rho((0,1 / x)),\right.
$$

\footnotetext{
${ }^{2}$ See, e.g., Families $3,5,27,28,29,33,35,45,46,63,64,88$, and 89 in the list of complete Bernstein functions of Schilling et al. (2010, Chapter 15).
} 
where $g_{\rho}^{-1}$ denotes the generalized inverse of the non-increasing function $g_{\rho}$.

(c) We have the following equality in law, with $\left\{\epsilon_{k}, J_{k}\right\}_{k \geq 1}$ iid unit exponentials:

$$
H_{1} \stackrel{d}{=} \sum_{k \geq 1} J_{k} g_{\rho}^{-1}\left(\epsilon_{1}+\ldots+\epsilon_{k}\right) \boldsymbol{1}_{\left\{\epsilon_{1}+\ldots+\epsilon_{k} \leq \rho((0, \infty))\right\}} .
$$

Proof: $\quad$ (a) We compute, using (4.1), $\Psi_{L}(x)=x /(x+1)$, and (4.3) that

$$
\begin{aligned}
\Psi_{H}(x) & =\int_{0}^{\infty} \Psi_{L}(x u) \nu_{F}(\mathrm{~d} u)=\int_{0}^{\infty} \frac{x u}{x u+1} \nu_{F}(\mathrm{~d} u) \\
& =\int_{0}^{\infty} \frac{x}{x+\frac{1}{u}} \nu_{F}(\mathrm{~d} u)=\int_{0}^{\infty} \frac{x}{x+u} \rho_{F}(\mathrm{~d} u) .
\end{aligned}
$$

(b) We recall from Lemma 2.2 that $F \mapsto \nu_{F}$ is a bijection between $\hat{\mathfrak{F}}$ and Lévy measures on $(0, \infty)$. Furthermore, it is clear that the mapping $\nu_{F} \mapsto \rho_{F}$ is injective. Left to show is only that every Stieltjes measure is attainable, i.e. that $\nu_{F} \mapsto \rho_{F}$ is surjective. To this end, it is sufficient to show for an arbitrary Stieltjes measure $\rho$ that the measure

$$
\nu(A):=\rho\left(\left\{\frac{1}{u}: u \in A\right\}\right), \quad A \subset(0, \infty) \text { a Borel set, }
$$

is a Lévy measure on $(0, \infty)$. To this end, we observe that

$$
\begin{aligned}
\nu((1, \infty)) & =\rho((0,1)) \leq \int_{0}^{1} \frac{2}{1+t} \rho(\mathrm{d} t) \leq 2 \int_{0}^{\infty} \frac{1}{1+t} \rho(\mathrm{d} t)<\infty \\
\int_{0}^{1} u \nu(\mathrm{d} u) & =\int_{1}^{\infty} \frac{1}{t} \rho(\mathrm{d} t) \leq \int_{1}^{\infty} \frac{2}{t+1} \rho(\mathrm{d} t) \leq 2 \int_{0}^{\infty} \frac{1}{t+1} \rho(\mathrm{d} t)<\infty .
\end{aligned}
$$

The claimed expression for $F_{\rho}$ can be retrieved directly from Lemma 2.2, while noticing that $\rho_{F}((0,1 / x))=\nu_{F}((x, \infty))$.

(c) This is a direct consequence of part (b) and the definition of the process $H$.

Like the series representation in Example 4.1 for ID $[0, \infty]$ is useful if the Lévy measure is nice, the representation in Lemma 4.2 (c) can be used to construct simulation algorithms for infinitely divisible distributions from the Bondesson family, when the Stieltjes measure $\rho$ is nice. In case of a compound Poisson distribution the series is even finite, hence the simulation algorithm is exact. We provide some examples to demonstrate this procedure.

Example 4.3 (Exact simulation of compound Poisson laws). Let $\rho$ be some finite measure on $(0, \infty)$, hence it automatically is a Stieltjes measure. We denote by $X$ a generic random variable in $\operatorname{BO}(0, \infty)$ associated with this Stieltjes measure. There exists a distribution function $G$ of some non-negative random variable with $G(0)=0$ and a constant $\beta:=\rho((0, \infty))>0$ such that $\rho((0, x])=\beta G(x)$. The Lévy measure $\nu$ associated with this Stieltjes measure $\rho$ is determined by its survival function, which satisfies $\nu((x, \infty))=\beta \varphi_{G}(x)$, where $\varphi_{G}(x):=\beta \int_{0}^{\infty} \exp (-x u) \mathrm{d} G(u)$ denotes the Laplace transform of $\mathrm{d} G$. We observe

$$
g_{\rho}^{-1}(y)=\frac{1}{G^{-1}(y / \beta)}, \quad 0<y<\beta,
$$


where $G^{-1}$ is the generalized inverse of the distribution function $G$. Consequently, according to Lemma 4.2(c) the compound Poisson distribution $X$ with intensity $\beta$ and jump size density $-\varphi_{G}^{\prime}$ has the finite series representation

$$
X \stackrel{d}{=} \sum_{k \geq 1} \frac{J_{k}}{G^{-1}\left(\left(\epsilon_{1}+\ldots+\epsilon_{k}\right) / \beta\right)} \mathbf{1}_{\left\{\epsilon_{1}+\ldots+\epsilon_{k} \leq \beta\right\}}
$$

with $\left\{\epsilon_{k}, J_{k}\right\}_{k>1}$ iid unit exponentials. It is not difficult to come up with examples for $G$ such that $G^{-1}$ is in closed form, but neither is a simulation algorithm for the density $-\varphi_{G}^{\prime}$ at hand, nor is the inverse of $\varphi_{G}$ in closed form. In such a situation, (4.4) provides a convenient basis for an exact simulation algorithm. One particular example is given by Family 45 in Schilling et al. (2010, Chapter 15): we have $\beta=0.5$ and $G(x)=\min \{x, 1\}(2-\min \{x, 1\})$ and obtain $G^{-1}(x)=1-\sqrt{1-x}$ for $x \in(0,1)$, leading to

$$
X \stackrel{d}{=} \sum_{k \geq 1} \frac{J_{k} \mathbf{1}_{\left\{\epsilon_{1}+\ldots+\epsilon_{k} \leq 0.5\right\}}}{1-\sqrt{1-2\left(\epsilon_{1}+\ldots+\epsilon_{k}\right)}} .
$$

The associated Bernstein function is given by $\Psi(x)=x(1+x) \log (1+1 / x)-x$.

Example 4.4 (A few non-compound Poisson examples). With a parameter $\theta>0$, we consider the complete, unbounded Bernstein functions

$$
\Psi_{1}(x)=\frac{x}{\sqrt{x+\theta}}, \quad \Psi_{2}(x)=\frac{x}{\theta-x} \log \left(\frac{\theta}{x}\right), \quad \Psi_{3}(x)=\sqrt{x} \arctan \left(\sqrt{\frac{x}{\theta}}\right),
$$

which correspond to Families 5, 33, and 64 in Schilling et al. (2010, Chapter 15). For each of them, the associated Stieltjes measure has a convenient form, namely

$$
\rho_{1}(\mathrm{~d} x)=\frac{\mathbf{1}_{\{x>\theta\}} \mathrm{d} x}{\pi \sqrt{x-\theta}}, \quad \rho_{2}(\mathrm{~d} x)=\frac{\mathrm{d} x}{\theta+x}, \quad \rho_{3}(\mathrm{~d} x)=\frac{\mathbf{1}_{\{x>\theta\}} \mathrm{d} x}{2 \sqrt{x}},
$$

and the associated function $g_{\rho}^{-1}$ from Lemma 4.2 can be computed in closed form, to wit

$$
g_{\rho_{1}}^{-1}(x)=\frac{1}{\theta+(\pi x / 2)^{2}}, \quad g_{\rho_{2}}^{-1}(x)=\frac{1}{\theta\left(e^{x}-1\right)}, \quad g_{\rho_{3}}^{-1}(x)=\frac{1}{(x+\sqrt{\theta})^{2}} .
$$

\section{Conclusion}

We have studied a semi-parametric family of non-decreasing stochastic processes $H=\left\{H_{t}\right\}_{t \geq 0}$, which comprises (possibly killed) Lévy subordinators without drift as a special case. Whereas a Lévy subordinator $L$ is conveniently specified by a Bernstein function $\Psi_{L}$, the process $H$ is specified by a pair $\left(\Psi_{F}, \Psi_{L}\right)$ of two Bernstein functions. From a theoretical point of view, we have demonstrated that the parameterization in terms of a distribution function $F$ and a Lévy subordinator $L$ provides a convenient apparatus to study distributional properties of $H$. In particular, we have established a canonical series representation and have studied the natural filtration of $H$, highlighting that the independent increment property is exclusive to the Lévy subordinator subfamily. From a practical point of view, we have explained how to simulate exactly and accurately the $d$-variate extreme-value copula associated with each process $H$. Furthermore, we have used the derived setting to establish a series representation for infinitely divisible laws from the Bondesson family that is given in terms of its Stieltjes measure only. 


\section{Acknowledgments}

We like to thank an anonymous referee for valuable feedback on an earlier version of the manuscript. In particular, his or her suggestions helped us to streamline and revise some of the proofs, in particular Formula (1.3) and Lemma 2.14. Of course, all remaining errors are ours.

\section{References}

A. Behme. Exponential functionals of Lévy processes with jumps. ALEA Lat. Am. J. Probab. Math. Stat. 12 (1), 375-397 (2015). MR3368963.

C. Bender and T. Marquardt. Integrating volatility clustering into exponential Lévy models. J. Appl. Probab. 46 (3), 609-628 (2009). MR2560892.

G. Bernhart, J.-F. Mai and M. Scherer. On the construction of low-parametric families of min-stable multivariate exponential distributions in large dimensions. Depend. Model. 3 (1), 29-46 (2015). MR3418655.

J. Bertoin. Subordinators: examples and applications. In Lectures on probability theory and statistics (Saint-Flour, 1997), volume 1717 of Lecture Notes in Math., pages 1-91. Springer, Berlin (1999). MR1746300.

L. Bondesson. Classes of infinitely divisible distributions and densities. Z. Wahrsch. Verw. Gebiete 57 (1), 39-71 (1981). MR623454.

L. Bondesson. On simulation from infinitely divisible distributions. Adv. in Appl. Probab. 14 (4), 855-869 (1982). MR677560.

C. M. Cuadras and J. Augé. A continuous general multivariate distribution and its properties. Comm. Statist. A-Theory Methods 10 (4), 339-353 (1981). MR612401.

C. Dombry, S. Engelke and M. Oesting. Exact simulation of max-stable processes. Biometrika 103 (2), 303-317 (2016). MR3509888.

K. Es-sebaiy and Y. Ouknine. How rich is the class of processes which are infinitely divisible with respect to time? Statist. Probab. Lett. 78 (5), 537-547 (2008). MR2400867.

J. Galambos. Order statistics of samples from multivariate distributions. J. Amer. Statist. Assoc. 70 (351, part 1), 674-680 (1975). MR0405714.

J. P. Gilman, I. Kra and R. E. Rodríguez. Complex analysis, volume 245 of Graduate Texts in Mathematics. Springer, New York (2007). ISBN 978-0-387-74714-9. MR2370126.

A. Gnedin and J. Pitman. Regenerative composition structures. Ann. Probab. 33 (2), 445-479 (2005). MR2122798.

A. Gnedin and J. Pitman. Moments of convex distribution functions and completely alternating sequences. In Probability and statistics: essays in honor of David A. Freedman, volume 2 of Inst. Math. Stat. (IMS) Collect., pages 30-41. Inst. Math. Statist., Beachwood, OH (2008). MR2459948.

G. Gudendorf and J. Segers. Extreme-value copulas. In Copula theory and its applications, volume 198 of Lect. Notes Stat. Proc., pages 127-145. Springer, Heidelberg (2010). MR3051266.

E. J. Gumbel. Bivariate exponential distributions. J. Amer. Statist. Assoc. 55, 698-707 (1960). MR0116403.

E. J. Gumbel. Bivariate logistic distributions. J. Amer. Statist. Assoc. 56, 335-349 (1961). MR0158451. 
L. de Haan and S. I. Resnick. Limit theory for multivariate sample extremes. Z. Wahrscheinlichkeitstheorie und Verw. Gebiete 40 (4), 317-337 (1977). MR0478290.

A. Hakassou and Y. Ouknine. IDT processes and associated Lévy processes with explicit constructions. Stochastics 85 (6), 1073-1111 (2013). MR3176501.

L. F. James, B. Roynette and M. Yor. Generalized gamma convolutions, Dirichlet means, Thorin measures, with explicit examples. Probab. Surv. 5, 346-415 (2008). MR2476736.

L. F. James and Z. Zhang. Quantile clocks. Ann. Appl. Probab. 21 (5), 1627-1662 (2011). MR2884047.

H. Joe. Multivariate models and dependence concepts, volume 73 of Monographs on Statistics and Applied Probability. Chapman \& Hall, London (1997). ISBN 0-412-07331-5. MR1462613.

C. Kopp and I. Molchanov. Series representation of time-stable stochastic processes. Probab. Math. Statist. 38 (2), 299-315 (2018). MR3896713.

J.-F. Mai. Extreme-value copulas associated with the expected scaled maximum of independent random variables. J. Multivariate Anal. 166, 50-61 (2018). MR3799634.

J.-F. Mai. Canonical spectral representation for exchangeable max-stable sequences. Extremes (2019). DOI: 10.1007/s10687-019-00361-3.

J.-F. Mai and M. Scherer. Lévy-frailty copulas. J. Multivariate Anal. 100 (7), 1567-1585 (2009). MR2514148.

J.-F. Mai and M. Scherer. Reparameterizing Marshall-Olkin copulas with applications to sampling. J. Stat. Comput. Simul. 81 (1), 59-78 (2011). MR2747378.

J.-F. Mai and M. Scherer. Characterization of extendible distributions with exponential minima via processes that are infinitely divisible with respect to time. Extremes 17 (1), 77-95 (2014). MR3179971.

J.-F. Mai and M. Scherer. Simulating copulas, volume 6 of Series in Quantitative Finance. World Scientific Publishing Co. Pte. Ltd., Hackensack, NJ (2017). ISBN 978-981-3149-24-3. MR3729417.

R. Mansuy. On processes which are infinitely divisible with respect to time. ArXiv Mathematics e-prints (2005). Working paper. arXiv: math/0504408.

A. W. Marshall and I. Olkin. A multivariate exponential distribution. J. Amer. Statist. Assoc. 62, 30-44 (1967). MR0215400.

R. B. Nelsen. An introduction to copulas. Springer Series in Statistics. Springer, New York, second edition (2006). ISBN 978-0387-28659-4; 0-387-28659-4. MR2197664.

J. Pickands III. Multivariate extreme value distributions. In Proceedings of the 43rd session of the International Statistical Institute, Vol. 2 (Buenos Aires, 1981), volume 49, pages 859-878, 894-902 (1981). MR820979.

S. I. Resnick. Extreme values, regular variation, and point processes, volume 4 of Applied Probability. A Series of the Applied Probability Trust. Springer-Verlag, New York (1987). ISBN 0-387-96481-9. MR900810.

P. Ressel. Homogeneous distributions - and a spectral representation of classical mean values and stable tail dependence functions. J. Multivariate Anal. 117, 246-256 (2013). MR3053546.

R. L. Schilling, R. Song and Z. Vondraček. Bernstein functions, volume 37 of De Gruyter Studies in Mathematics. Walter de Gruyter \& Co., Berlin (2010). ISBN 978-3-11-021530-4. MR2598208. 
M. Schlather. Models for stationary max-stable random fields. Extremes 5 (1), 33-44 (2002). MR1947786. 